\title{
CFD Modeling and Control of a Steam Methane Reforming Reactor
}

\author{
Liangfeng Lao ${ }^{\mathrm{a}}$, Andres Aguirre ${ }^{\mathrm{a}}$, Anh Tran ${ }^{\mathrm{a}}$, Zhe $\mathrm{Wu}^{\mathrm{a}}$, Helen Durand ${ }^{\mathrm{a}}$, \\ Panagiotis D. Christofides ${ }^{*, a, b}$ \\ ${ }^{a}$ Department of Chemical and Biomolecular Engineering, University of California, Los Angeles, \\ CA, 90095-1592, USA. \\ ${ }^{b}$ Department of Electrical Engineering, University of California, Los Angeles, CA 90095-1592, \\ USA.
}

\begin{abstract}
This work initially focuses on developing a computational fluid dynamics (CFD) model of an industrial-scale steam methane reforming reactor (reforming tube) used to produce hydrogen. Subsequently, we design and evaluate three different feedback control schemes to drive the area-weighted average hydrogen mole fraction measured at the reforming tube outlet $\left(\bar{x}_{H_{2}}^{\text {outlet }}\right)$ to a desired set-point value $\left(\bar{x}_{H_{2}}^{\text {set }}\right)$ under the influence of a tube-side feed disturbance. Specifically, a CFD model of an industrial-scale reforming tube is developed in ANSYS Fluent with realistic geometry characteristics to simulate the transport and chemical reaction phenomena with approximate representation of the catalyst packing. Then, to realize the real-time regulation of the hydrogen production, the manipulated input and controlled output are chosen to be the outer reforming tube wall temperature profile and $\bar{x}_{H_{2}}^{\text {outlet }}$ respectively. On the problem of feedback control, a pro-
\end{abstract}

\footnotetext{
*Corresponding author: Tel: +1 (310) 794-1015; Fax: +1 (310) 206-4107; E-mail: pdc@seas.ucla.edu.
} 
portional (P) control scheme, a proportional-integral (PI) control scheme and a control scheme combining dynamic optimization and integral feedback control to generate the outer reforming tube wall temperature profile based on $\bar{x}_{H_{2}}^{\text {set }}$ are designed and integrated into real-time CFD simulation of the reforming tube to track $\bar{x}_{H_{2}}^{\text {set }}$. The CFD simulation results demonstrated that feedback control schemes can drive the value of $\bar{x}_{H_{2}}^{\text {outlet }}$ toward $\bar{x}_{H_{2}}^{\text {set }}$ in the presence of a tube-side feed disturbance, and can significantly improve the process dynamics compared to the dynamics under open-loop control.

Key words: Steam methane reforming, CFD modeling, process dynamics, process identification, process control, distributed parameter systems

\section{Introduction}

The steam methane reforming (SMR) process, which produces bulk hydrogen gas from methane through catalytic reactions, is the most common commercial 4 method for industrial hydrogen production. A general industrial-scale SMR pro-

cess can be described by the schematic of Fig. 11 The steam methane reformer (for simplicity, it is denoted as "reformer" in the following text) is the core unit in a SMR process which has a process (tube) side and a furnace side that interact through heat exchange through the walls of reforming reactors (for simplicity, they are denoted as the "reforming tubes"). In the furnace side, combustion of the furnace-side feed, usually a mixture of methane, hydrogen, carbon dioxide, carbon monoxide and air, heats the reforming tubes via radiative heat exchange; inside the reforming tubes, catalytic reactions take place, converting steam and methane into hydrogen and carbon oxides (including $\mathrm{CO}$ and $\mathrm{CO}_{2}$ ). A traditional 

tunnels transporting the flue gas out of the reformer, and reforming tubes.

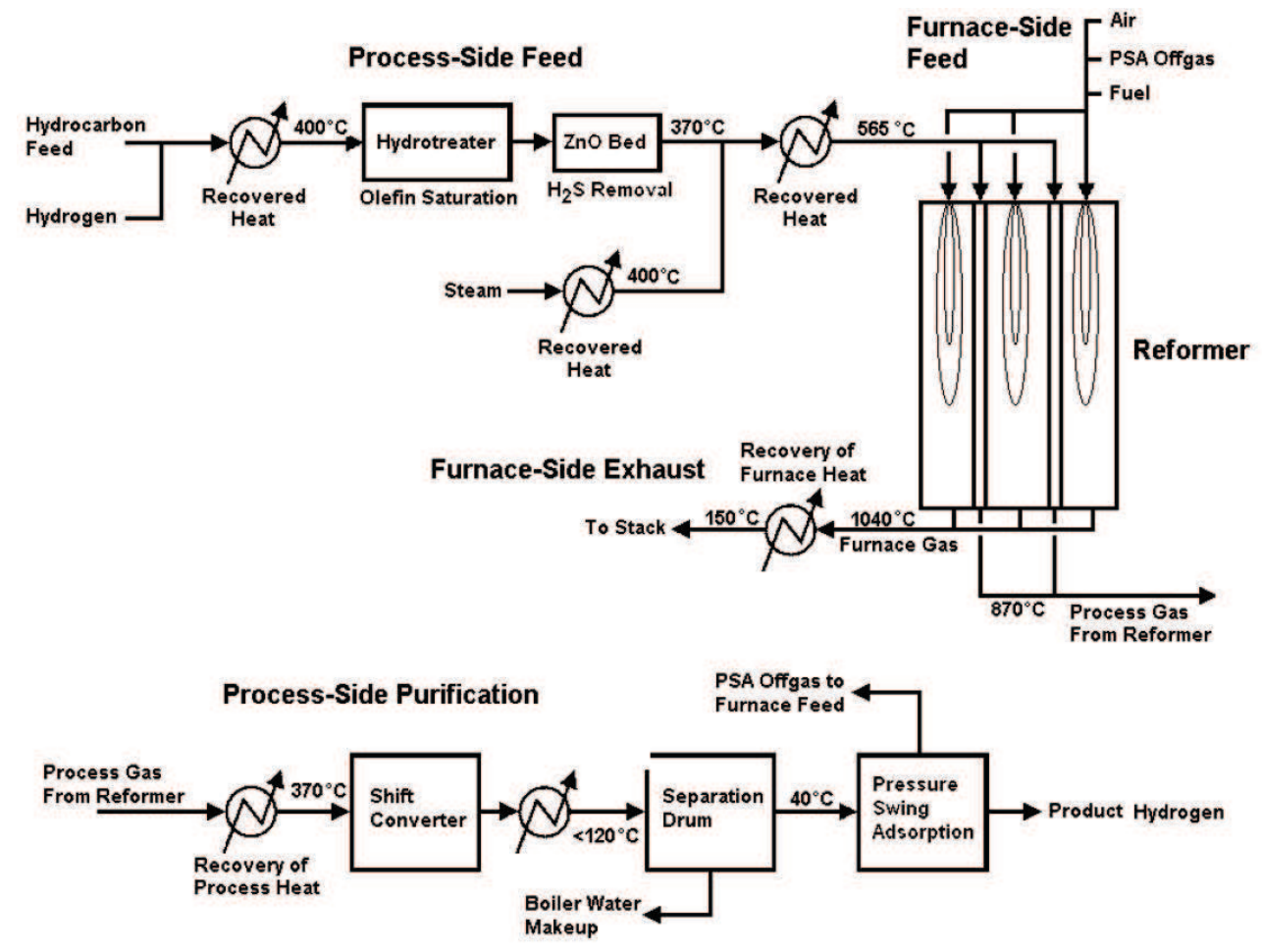

Figure 1: Steam methane reforming process diagram Othmer. furnace-side feed, refractory walls enveloping the combustion products, flue gas

top-fired, co-current furnace usually includes top burners which are fed with the

\section{(1)}

of 9 (19) ena inside the reformer, more comprehensive mathematical models have been developed considering more detailed and precise radiation mechanisms, combus- 
tion models, flue gas flow patterns, SMR reaction kinetics and packed bed reactor models Latham (2008). However, solving these complete reformer models is computationally expensive due to the increasing complexity of the fundamental nonlinear partial differential equations describing reformer physico-chemical phenomena. Also, for large reformers with complicated geometry, the geometry characteristics and various boundary conditions Latham (2008) make mathematical modeling very difficult.

On the other hand, with the dramatic increase of computing power, computational fluid dynamics (CFD) modeling has become an increasingly important platform for reformer modeling and design, combining physical and chemical models with detailed representation of the reformer geometry. When compared with first-principles modeling, CFD is a modeling technique with powerful visualization capabilities to deal with various geometry characteristics and boundary conditions. Moreover, CFD modeling provides flexibility to modify design parameters without the expense of hardware changes which brings large economic and time savings Uriz et al. (2013). CFD technology has been successful in carrying out the simulation of industrial furnaces Baburić et al. (2005); Han et al. (2006); Stefanidis et al. (2006); Noor et al. (2013) and SMR tube reactors, i.e., reforming tubes modeled as packed-bed reactors Calis et al. (2001); Behnam et al. (2012); Dixon (2014); Guardo et al. (2004). Specifically, recent attempts to use CFD modeling to characterize the physico-chemical phenomena of transport and reaction processes inside reforming tubes have been done exclusively on a microscopic or bench-scale level, e.g., the effect of catalyst orientation on catalytic 
performance is investigated with a CFD model of a single catalyst particle Dixon (2014), and the validation of CFD simulation results to experimental data is performed with a CFD model of a bench-scale reforming tube Behnam et al. (2012). In the present work, we focus on an industrial-scale reforming tube, i.e, the external diameter, internal diameter and exposed length of the reforming tube are $14.6 \mathrm{~cm}, 12.6 \mathrm{~cm}$ and $12.5 \mathrm{~m}$ respectively. It is noteworthy that the CFD model of the industrial-scale reforming tube in this work is developed based on a unit currently employed in a commercial plant. Therefore, the CFD model has the geometry characteristics of an industrial reforming tube, i.e., the length and inner and outer tube radii, includes an industrially relevant representation of the catalyst network, and incorporates appropriate boundary conditions including the reforming tube outer wall temperature and tube-side feed conditions. A major purpose of the CFD modeling in this work, in addition to its purpose as a means for evaluating various control strategies, is to propose a method of industrial reforming tube modeling with considerable modeling accuracy that can help evaluate various modeling assumptions usually employed.

The production rate of hydrogen fuel from a typical SMR process strongly depends on the operating temperature of the furnace, where the aforementioned reforming tubes are encapsulated, and more specifically the outer reforming tube wall temperature. Because of the endothermic nature of SMR reactions, a higher outer reforming tube wall temperature theoretically results in a higher production rate of hydrogen fuel. On the other hand, operating the reforming tubes at excessively high temperature can lead to disastrous consequences and significant 
capital loss. Particularly, the formation of carbon on the catalyst surface and on the inner reforming tube surface prevents the reactants from entering the catalyst active sites and reduces the rate of heat transfer to the tube-side gas mixture respectively. As a result, the reaction progress and hydrogen production might be disrupted. Additionally, the expected lifetime of reforming tubes is extremely sensitive to changes in operating temperatures, i.e., an increase in tube wall temperature of $20 \mathrm{~K}$ can reduce the tube lifetime by half Pantoleontos et al. (2012) Latham (2008). Moreover, reforming tubes are one of the most expensive plant components as they account for approximately $10 \%$ of the capital cost of an entire hydrogen plant Latham (2008), and the total replacement of a typical industrial-scale reformer is expected to cost $5-8$ million USD Pantoleontos et al. (2012). Consequently, the outer reforming tube wall temperature is required to be closely monitored and kept slightly below the design tube wall temperature in industrial standard operation in order to eliminate the potential stress-to-rupture of tube materials Beyer et al. (2005). Through CFD simulation, these above critical issues can be detected and predicted, and corresponding changes can be applied to improve the reforming tube design and operating parameters.

Motivated by the above considerations, we initially develop a CFD model of an industrial-scale reforming tube in ANSYS Fluent with realistic tube geometry characteristics to simulate the transport and reaction phenomena with an approximate representation of the catalyst packing inside the reforming tube. Next, we utilize publicly available SMR plant data to construct the proper boundary conditions for the reforming tube inlet, the reforming tube outlet and the outer reform- 
ing tube wall, so that the simulation results and the available industrial plant data are consistent Latham (2008). Due to the high Reynolds number of the tube-side gas mixture, significant pressure gradients across the reforming tube and wellacknowledged radial gradients near the reforming tube inlet, the standard $k-\epsilon$ turbulence model with enhanced wall treatment is implemented. Furthermore, an intrinsic SMR reaction kinetic model Xu and Froment (1989), widely accepted in academia and industry, is used to derive the chemical conversion rate equations, which account for the effect of internal and external diffusion limitations on the observed rates as well as for the presence of the catalyst particles inside the reforming tube. Subsequently, to adjust the hydrogen production of the reforming tube in real-time, we propose the design and implementation of feedback control schemes into the CFD model. Specifically, the manipulated input and controlled output are chosen to be the outer reforming tube wall temperature and the areaweighted average hydrogen mole fraction measured at the reforming tube outlet $\left(\bar{x}_{H_{2}}^{\text {outlet }}\right)$ respectively, and the objective is to drive $\bar{x}_{H_{2}}^{\text {outlet }}$ to the desired set-point $\left(\bar{x}_{H_{2}}^{\text {set }}\right)$ under the influence of a tube-side feed disturbance. On the problem of feedback control, firstly, a proportional (P) control scheme and a proportionalintegral (PI) control scheme generating the outer reforming tube wall temperature trajectory based on the desired $\bar{x}_{H_{2}}^{\text {set }}$ are designed to realize the closed-loop CFD simulation. Finally, motivated by industrial concerns, we also design a feedback control scheme combining dynamic optimization and integral feedback control. The control performance of these three control schemes is evaluated with respect to tracking the set-point, improving the speed of the closed-loop responses and 
compensating for the effect of the tube-side feed disturbance on $\bar{x}_{H_{2}}^{\text {outlet }}$.

\section{Single Reforming Tube Model}

\subsection{Industrial Level Geometry}

The reforming tube investigated in this work belongs to an industrial-scale top-fired, co-current reformer designed by Selas Fluid Processing Corporation (Figs. 2, 3). The furnace of this reformer contains seven rows of forty-eight reforming tubes whose external diameter, internal diameter and exposed length are $14.6 \mathrm{~cm}, 12.6 \mathrm{~cm}$ and $12.5 \mathrm{~m}$ respectively. Each reforming tube is tightly packed with specially designed nickel oxide over alpha alumina support (i.e., $\mathrm{Ni} / \alpha-\mathrm{Al}_{2} \mathrm{O}_{3}$ ) catalyst particles, which not only facilitate the formation of hydrogen fuel from steam and methane through the highly endothermic SMR reactions, but also play a role as an intermediate medium to enhance the rate of heat transfer to the tube-side gas mixture. These rows of tubes are separated by eight rows of twelve burners (Fig. 2) which are fed with the furnace-side gas composed of three parts, i.e., natural gas, combustion air $\left(\mathrm{Ar}, \mathrm{N}_{2}\right.$ and $\left.\mathrm{O}_{2}\right)$ and tail gas $\left(\mathrm{CO}\right.$ and $\left.\mathrm{H}_{2}\right)$. Based on the composition of a typical furnace-side gas that is given in Table 1, the combustion is a fuel lean process so that the fuel can be completely combusted over a flame length of $4.5-6 m$ releasing the thermal energy needed to drive the highly endothermic SMR reactions Latham (2008). The thermal energy released by the combustion of furnace fuel is transferred to the reforming tubes predominantly by radiation inside the high-temperature furnace chamber. At the bottom of the furnace, the rows of tubes are separated by the rectangular intrusions known as flue gas tunnels or coffin boxes (Fig. 3). The flue gas tunnels extend from the 
front to the back of the furnace with a height of $2.86 \mathrm{~m}$ from the floor and allow the furnace flue gas to exit the furnace. Thirty-five extraction ports are distributed in a row along the sides of each flue gas tunnel. The furnace flue gas enters the tunnels from the furnace chamber through the extraction ports and then exits the furnace through the front openings of the coffin boxes. It is worth noting that the dynamics of all aforementioned components are tightly coupled inside the furnace during operation.

Under ideal operating conditions, the environments surrounding all reforming tubes of the SMR furnace unit are very similar, such that it is necessary to model only a single reforming tube to characterize the conditions in all 336 reforming tubes. Thus, the scope of the modeling part in this work only aims to develop a CFD model with realistic dimensions, geometry and characteristics of one industrial-scale steam methane reforming tube, and in this regard, we develop and evaluate all essential simulation settings of the reforming tube model to accomplish the modeling objective in the following sections. By focusing on one reforming tube, we are able to accomplish the primary objective of this work, which is presented later, and which is to demonstrate that real-time feedback control schemes can be incorporated into the CFD model of an industrial-scale reforming tube to build closed-loop systems that have desired dynamic response behavior and produce a desired amount of hydrogen.

\subsection{Tube Geometry and Meshing}

Mesh quality is the most critical issue for accurate and successful CFD modeling, i.e., a low quality mesh requires the most robust CFD solver and significantly 
Table 1: Furnace-side inlet operating conditions.

\begin{tabular}{ll}
\hline Pressure $(\mathrm{kPa})$ & 132.4 \\
\hline Temperature $(\mathrm{K})$ & 524 \\
\hline Flow rate $(\mathrm{kg} / \mathrm{s})$ & 1.08 \\
\hline$x_{\mathrm{CH}}$ & 0.0517 \\
\hline$x_{\mathrm{H}_{2} 0}$ & 0.0306 \\
\hline$x_{\mathrm{CO}}$ & 0.0211 \\
\hline$x_{\mathrm{H}_{2}}$ & 0.0540 \\
\hline$x_{\mathrm{O}_{2}}$ & 0.1530 \\
\hline$x_{A r}$ & 0.0077 \\
\hline$x_{N_{2}}$ & 0.5793 \\
\hline
\end{tabular}

greater computing resources to determine a converged solution. Due to the axisymmetric geometry property of the reforming tube as shown in Fig. 4(a), a twodimensional $(2 D)$ axisymmetric reforming tube geometry and its corresponding mesh structure were employed. The $2 D$ axisymmetric reforming tube mesh which is shown in Fig. 4(b) was constructed in the meshing software ICEM-CFD. The $2 D$ axisymmetric reforming tube mesh only contains 24690 quadrilateral cells, with a $100 \%$ orthogonal quality. Also, the CFD simulation of the single reforming tube only performs calculations for half of the tube mesh considering its $2 D$ axisymmetric properties. Consequently, this $2 \mathrm{D}$ axisymmetric reforming tube mesh brings large computational efficiency over a three-dimensional reforming tube CFD model.

In terms of mesh size of this single reforming tube, open-loop simulation results of CFD models with different mesh sizes indicate that 24690 cells are enough to represent this specific tube, and that the further increase of the mesh 


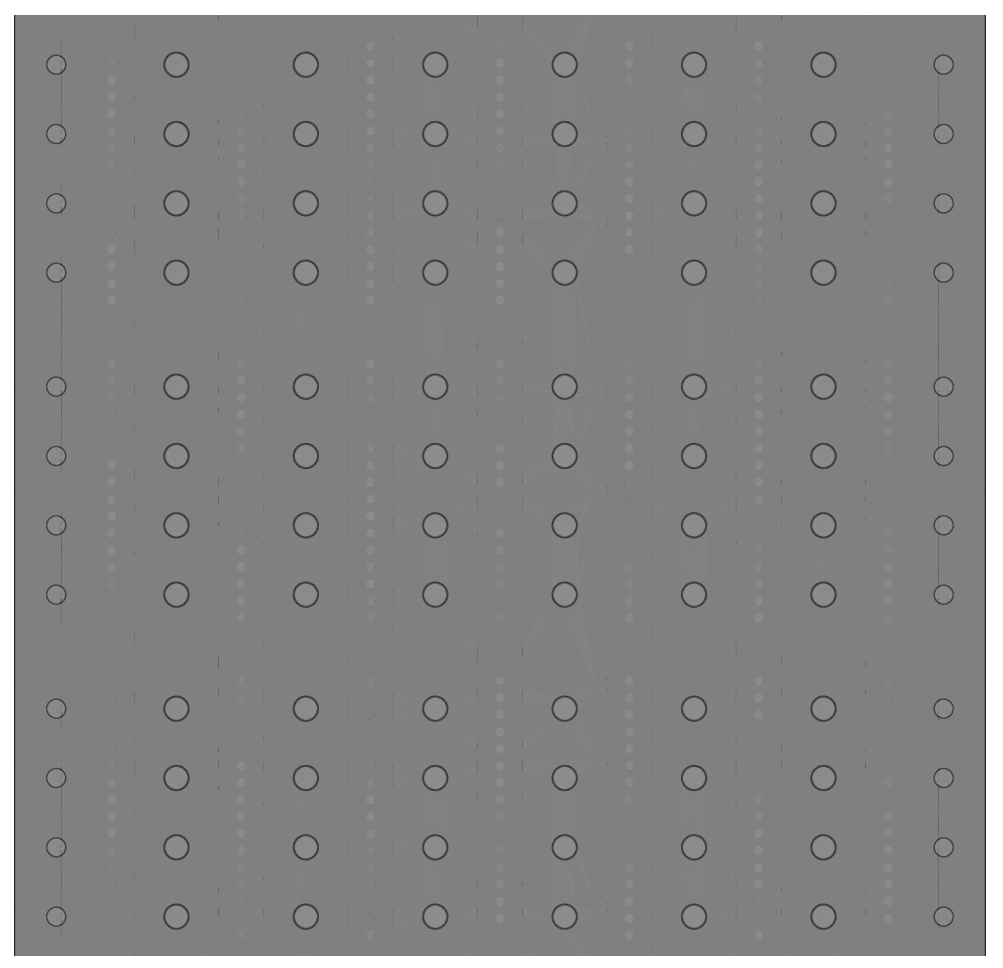

Figure 2: The top view of an industrial-scale top-fired, co-current reformer with 336 reforming tubes, for which each reforming tube is denoted by a smaller circle, and 96 burners, for which each burner is denoted by a larger circle.

size achieves identical results but requires significantly higher computation time.

For this reforming tube CFD model, boundary layer design is very critical to the modeling of the heat convection from the inner reforming tube wall to the tube-side gas mixture and the heat conduction from the inner reforming tube wall to the catalyst particles. To calculate the first node height from the inner reforming tube wall for the boundary layer design, NASA's Viscous Grid Spacing Calculator Aeronautics and Administration is adopted based on a suitable $Y^{+}$value Inc. (Nov. 2013a). For this specific single reforming tube geometry, five nodes are ap- 


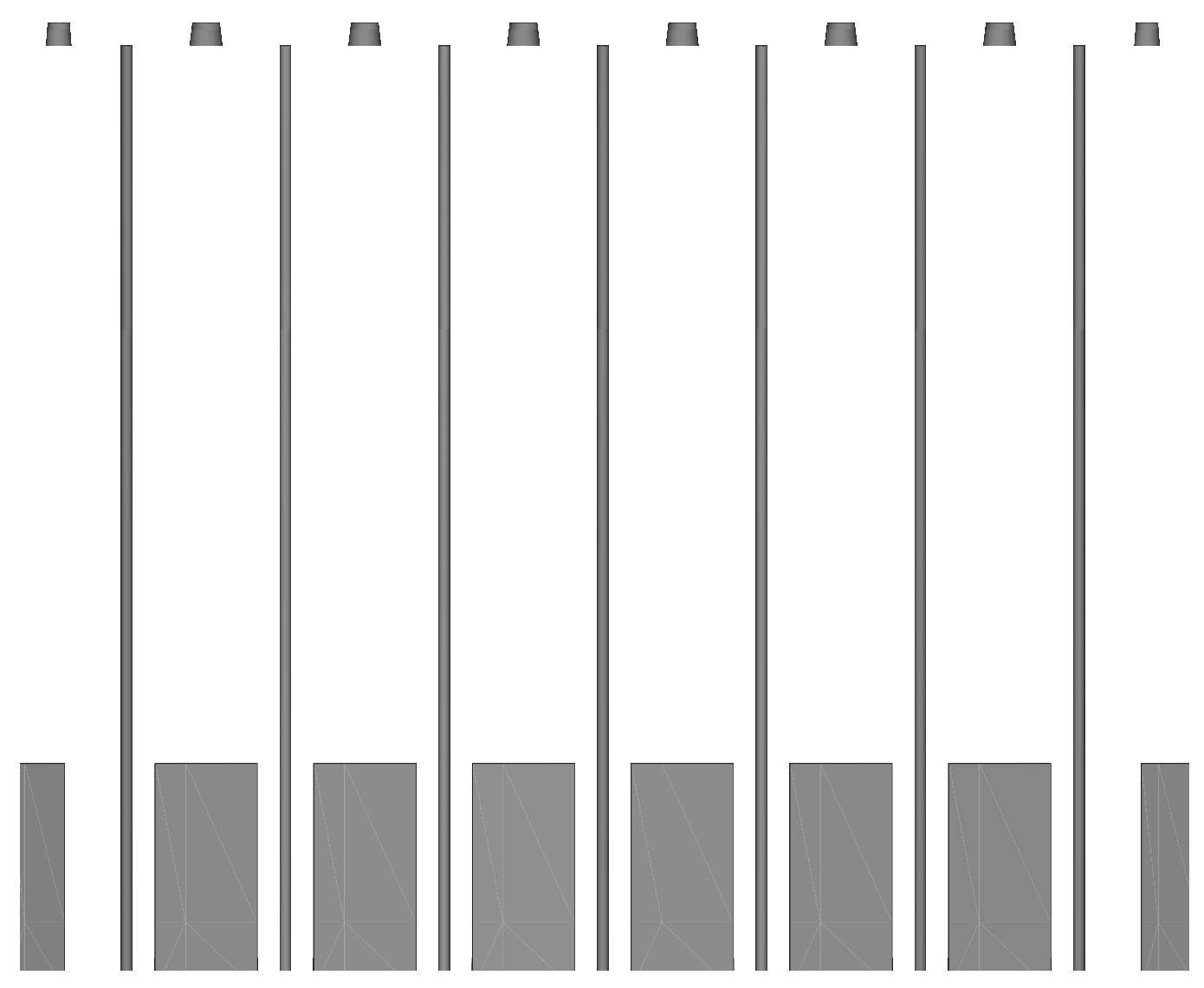

Figure 3: The front view of an industrial-scale top-fired, co-current reformer. It is important to note that the refractory wall is modeled to be transparent, so that the interior components can be seen. Specifically, eight rectangular boxes located on the floor represent the flue gas tunnels, eight frustums of cones located on the ceiling represent the corresponding rows of burners and seven slender rectangles connecting the ceiling and floor represent the corresponding rows of reforming tubes.

plied in the boundary layer at the inner reforming tube wall as requested by the two-equation $k-\epsilon$ turbulence model with the enhanced wall treatment function 
the boundary layer design is demonstrated in Fig. 4(b), We note here that NASA's Viscous Grid Spacing Calculator Aeronautics and Administration uses fixed viscosity, pressure and temperature values for the fluid properties which may not be suitable for most SMR calculations. Through a decompiling of the calculator, a revised algorithm based on the original calculator is developed for our specific inlet conditions of the tube-side gas mixture.

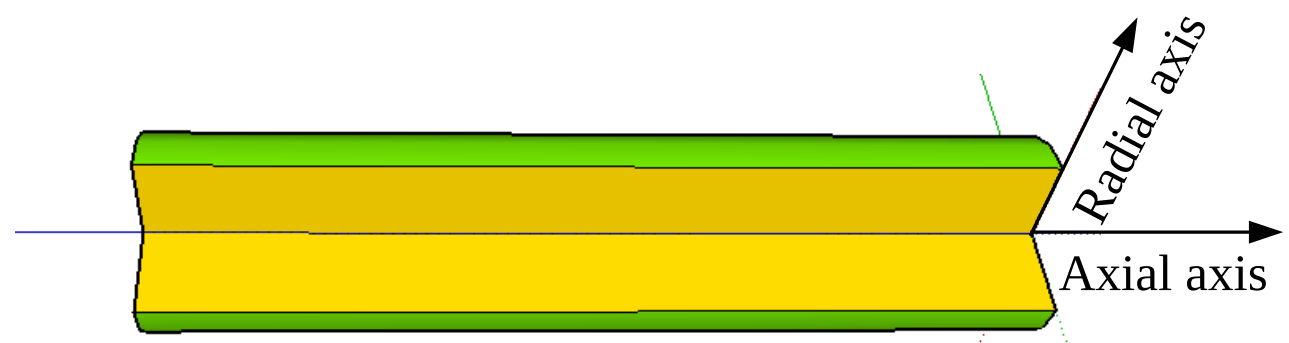

(a)

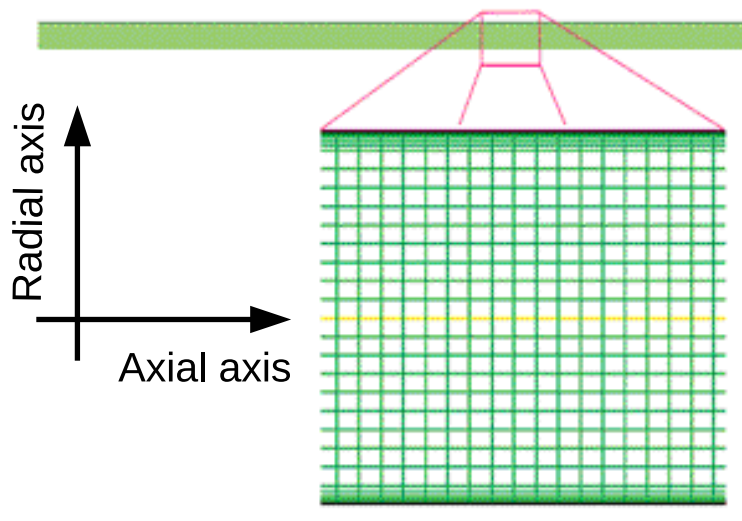

(b)

Figure 4: Two-dimensional axisymmetric reforming tube geometry (Fig.4(a)] and mesh structure (Fig. 4(b)). 


\subsection{Kinetic Model}

The conversion rates of reactants, i.e., steam and methane, into products, i.e., hydrogen fuel, and the direction of the reforming reactions and the water-gas shift reaction under different conditions (reactant concentration, temperature and pressure) must be accurately accounted for by using a reaction kinetics model. In a reforming tube, the reforming reactions and water-gas shift reaction occur at the catalyst active sites. In particular, reactants need to diffuse from the bulk tube-side gas mixture to the surface of the catalyst particle and then into the catalyst pores; after the formation of the products, products need to desorb from the catalyst cores and reenter the bulk tube-side gas mixture. However, a reaction kinetics model that provides a detailed treatment of these catalyst-specific phenomena would be unnecessarily complex. Additionally, the catalyst particles and the detailed packing pattern inside an industrial-scale packed-bed reactor are not explicitly modeled in this study.

To deal with this issue, in this single reforming tube CFD model, an intrinsic SMR reaction model $\mathrm{Xu}$ and Froment (1989) is used to describe the reactions happening inside the reforming tube. The SMR reaction kinetics are given in Eq. 1 below which is widely adopted in both CFD modeling and mathematical modeling of reforming tubes: 


$$
\begin{aligned}
& C H_{4}(g)+H_{2} O(g) \rightleftarrows C O(g)+3 H_{2}(g), \\
& r_{1}=\frac{k_{1}}{p_{H_{2}}^{2.5}}\left(p_{C H_{4}} p_{H_{2} O}-\frac{p_{H_{2}}^{3} p_{C O}}{K_{1}}\right) / D E N^{2} \\
& C O(g)+H_{2} O(g) \rightleftarrows C O_{2}(g)+H_{2}(g), \\
& r_{2}=\frac{k_{2}}{p_{H_{2}}}\left(p_{C O} p_{H_{2} O}-\frac{p_{H_{2}} p_{C O_{2}}}{K_{2}}\right) / D E N^{2} \\
& C H_{4}(g)+2 H_{2} O(g) \rightleftarrows C O_{2}(g)+4 H_{2}(g), \\
& r_{3}=\frac{k_{3}}{p_{H_{2}}^{3.5}}\left(p_{C H_{4}} p_{H_{2} O}^{2}-\frac{p_{H_{2}}^{4} p_{C O_{2}}}{K_{3}}\right) / D E N^{2} \\
& D E N=1+\frac{K_{H_{2} O} p_{H_{2} O}}{p_{H 2}} \\
& \quad+K_{C O} p_{C O}+K_{H_{2}} p_{H_{2}}+K_{C H_{4}} p_{C H_{4}}
\end{aligned}
$$

where $p_{\mathrm{H}_{2}}, p_{\mathrm{CH}_{4}}, p_{\mathrm{H}_{2} \mathrm{O}}, p_{\mathrm{CO}}$ and $p_{\mathrm{CO}_{2}}$ are the partial pressures of $\mathrm{H}_{2}, \mathrm{CH}_{4}, \mathrm{H}_{2} \mathrm{O}$, $\mathrm{CO}$ and $\mathrm{CO}_{2}$ respectively in the bulk tube-side gas mixture inside the reforming tube, $K_{H_{2}}, K_{C H_{4}}$ and $K_{C O}$ are adsorption constants for $H_{2}, \mathrm{CH}_{4}$ and $\mathrm{CO}$, respectively, $K_{\mathrm{H}_{2} \mathrm{O}}$ is a dissociative adsorption constant of $\mathrm{H}_{2} \mathrm{O}, k_{1}, k_{2}$ and $k_{3}$ are rate coefficients of the SMR reactions and $D E N$ is a dimensionless parameter. To realize this reaction kinetic model in CFD simulations by Fluent, each reversible reaction in Eq. 1 is split into two irreversible reactions. The reaction rates in Eq. 1 are implemented in Fluent by designing a user defined function (UDF) file. Since these intrinsic reaction kinetics do not consider the internal and external diffusion resistances of the catalyst particles, the reaction rates in Eq. 1 are multiplied by an effectiveness factor, 0.1 Wesenberg and Svendsen (2007), to account for the overall diffusion effects on the intrinsic reaction rates. 


\subsection{Compressible Gas Flow}

The tube-side inlet operating conditions of the reforming tubes are given in Table 2 Latham (2008). Based on the inlet conditions of the tube-side gas mixture, the Mach number is determined to be greater than 0.3 , and therefore, the density variations of the fluid flow due to high static pressure cannot be ignored. From the point of view of CFD simulation, when a pressure-based solver is used, like the one we chose for the simulation in this work, an accurate gas state equation is very critical to simulation accuracy. Based on this consideration, the compressible ideal gas state equation is adopted to describe the compressibility of the tube-side gas mixture.

Table 2: Process-side gas inlet operating conditions.

\begin{tabular}{ll}
\hline Pressure $(\mathrm{kPa})$ & 3038.5 \\
\hline Temperature $(\mathrm{K})$ & 887 \\
\hline Flow rate $(\mathrm{kg} / \mathrm{s})$ & 0.1161 \\
\hline$x_{\mathrm{CH}}$ & 0.2487 \\
\hline$x_{\mathrm{H}_{2} \mathrm{O}}$ & 0.7377 \\
\hline$x_{\mathrm{CO}}$ & 0.0001 \\
\hline$x_{\mathrm{H}_{2}}$ & 0.0018 \\
\hline$x_{\mathrm{CO}}$ & 0.0117 \\
\hline
\end{tabular}

234

\section{1} pro has (1)

A pressure-based solver that enables the pressure-based Navier-Stokes solution algorithm Inc. (Nov. 2013b) is chosen over a density-based solver. The pressure-based solver is more suited for a wider range of physical models and has features which are unavailable to the density-based solver, e.g., the physical velocity formulation for porous media which is adopted by this work to simulate 
the flow through the catalyst network in the reforming tube (this will be discussed later). It also provides more freedom for the simulations while converging to the same results obtained by the density-based solver.

\subsection{Porous Zone Design}

Table 3: Johnson Matthey's Katalco $23-4 Q$ catalyst properties.

\begin{tabular}{ll}
\hline Density, $\rho_{c}$ & $3960 \mathrm{~kg} / \mathrm{m}^{3}$ \\
\hline Heat Capacity, $C_{p, c}$ & $880 \mathrm{~J} /(\mathrm{kg} \cdot \mathrm{K})$ \\
\hline Thermal Conductivity, $k_{c}$ & $33 \mathrm{~W} /(\mathrm{m} \cdot \mathrm{K})$ \\
\hline Particle Diameter, $D_{p}$ & $3.5 \mathrm{~mm}$ (average) \\
\hline
\end{tabular}

Pressure drop is significant in industrial-scale reforming tubes when the tubeside gas mixture flows through the catalyst network made of many tightly packed catalyst particles. To estimate the pressure drop across the porous zone in the CFD modeling of turbulent flows, a semi-empirical expression, the Ergun equation Ergun and Orning (1949), which is applicable over a wide range of Reynolds numbers and for many packing patterns, is adopted as follows:

$$
\frac{\Delta P}{L}=\frac{150 \mu}{D_{p}^{2}} \frac{(1-\gamma)^{2}}{\gamma^{3}} v_{\infty}+\frac{1.75 \rho}{D_{p}} \frac{(1-\gamma)}{\gamma^{3}} v_{\infty}^{2}
$$

where $\Delta P$ is the pressure drop through the porous media, $L$ is the depth of the porous media, $\mu$ is the viscosity of the fluid, $\gamma$ is the porosity of the packed bed, $v_{\infty}$ is the bulk velocity of the fluid, $\rho$ is the density of the porous media, $\frac{150 \mu}{D_{p}^{2}} \frac{(1-\gamma)^{2}}{\gamma^{3}}$ is the viscous resistance coefficient and $\frac{1.75 \rho}{D_{p}} \frac{(1-\gamma)}{\gamma^{3}}$ is the inertial resistance coefficient. We assume that the viscous and inertial resistance coefficients are defined along the direction vectors $v_{1}=[1,0]$ (i.e., the principal axis direction) 
and $v_{2}=[0,1]$ (i.e., the radius direction) in the Cartesian two-dimensional $(2 D)$ coordinate system.

The modeling objective of this work is to develop a CFD model of an industrialscale reforming tube for a commercial plant. Thus, a common, readily available commercial catalyst, i.e., Johnson Matthey's Katalco 23-4Q Matthey also known as $\mathrm{Ni} / \alpha-\mathrm{Al}_{2} \mathrm{O}_{3}$, is chosen for this purpose, and its properties are shown in Table 3. It is noted that the intrinsic kinetic model used Xu and Froment (1989) was developed for a $\mathrm{Ni} / \mathrm{MgAl}_{2} \mathrm{O}_{4}$ catalyst, i.e., the catalyst support material is different than that utilized in this work. We considered the potential effects of the support material on the catalytic activity of the catalyst prior to selecting the kinetic model developed in Xu and Froment (1989) based on the work conducted by Jeong et al. (2006). First of all, the reduction peaks of both the $\mathrm{Ni} / \mathrm{MgAl}_{2} \mathrm{O}_{4}$ and $\mathrm{Ni} / \alpha-\mathrm{Al}_{2} \mathrm{O}_{3}$ catalyst types are in the neighborhood of $\sim 1083 \mathrm{~K}$, which means that they require similar heat input to become activated. Even though $\mathrm{Ni} / \mathrm{MgAl}_{2} \mathrm{O}_{4}$ is more active at lower temperatures than $\mathrm{Ni} / \alpha-\mathrm{Al}_{2} \mathrm{O}_{3}$, we speculate that both catalyst types have similar catalytic activity in our operating temperature range. Additionally, although the estimated surface area of $\mathrm{Ni} / \mathrm{MgAl}_{2} \mathrm{O}_{4}$ is higher than that of $\mathrm{Ni} / \alpha-\mathrm{Al}_{2} \mathrm{O}_{3}$ per unit mass of the catalyst, due to the large porosity of the packed bed inside the reforming tube, i.e., the void volume is approximately $60.9 \%$ of the reactor volume, and small inner reforming tube diameter of $0.126 \mathrm{~m}$, the mass of catalyst inside a single reforming tube is not significant. As a result, we decided to adopt the kinetic model developed in Xu and Froment (1989) in the CFD model to describe the chemistry inside the reforming tube us- 
ing the Johnson Matthey's Katalco $23-4 Q$ catalyst. Finally, the consistency of our simulation results in later sections with the data with which it is validated also demonstrates the effectiveness of the choice of the $\mathrm{Ni} / \alpha-\mathrm{Al}_{2} \mathrm{O}_{3}$ catalyst for the kinetic model based on a $\mathrm{Ni} / \mathrm{MgAl}_{2} \mathrm{O}_{4}$ catalyst. Additionally, from Table 2 and Table 3, the mass of the catalysts per reforming tube is $242 \mathrm{~kg}$, and the space-time of the tube is about 2080 seconds.

\subsection{Reforming Tube Wall Boundary Conditions}

For an industrial-scale reformer, the outer reforming tube wall temperature is usually in the range of $1100 \mathrm{~K}$ to $1178 \mathrm{~K}$ Latham (2008). In this single reforming tube simulation, to construct the temperature boundary condition of the outer reforming tube wall, the available plant data Latham (2008) are fit with a fourth order polynomial function by using a least squares linear regression method. The result of the fit shown in Fig. [5 is

$$
T_{\text {Wall }}(x)=\left[\begin{array}{lllll}
x^{4} & x^{3} & x^{2} & x^{1} & x^{0}
\end{array}\right]\left[\begin{array}{c}
-0.0221 \\
0.8003 \\
-10.734 \\
64.416 \\
997.16
\end{array}\right]
$$

where $T_{W a l l}(x)$ is the outer reforming tube wall temperature at a location $x(m)$ away from the reforming tube inlet, $x=0 \mathrm{~m}$. Using the boundary condition in Eq. 3, the reforming tube here is modeled as a heat sink, and thermal energy is transferred from the outer reforming tube wall to the inner reforming tube wall by heat conduction. The corresponding tube material properties are listed in Table 4 Davis (2000) which assumes that all the tube properties are temperature 
independent and the values at $T=1144 K$ are adopted in the CFD simulation. However, a constant outer reforming tube wall temperature profile is neither sufficient to maintain the current $\bar{x}_{H_{2}}^{\text {outlet }}$ when the reforming tube operating condition is subjected to a change in the tube-side mass flow rate (e.g., tube-side feed disturbance), nor capable of driving the current $\bar{x}_{H_{2}}^{\text {outlet }}$ to a new desired $\bar{x}_{H_{2}}^{\text {set }}$. To fulfill the overall scope of this work, which is to design and implement feedback control schemes to drive $\bar{x}_{H_{2}}^{\text {outlet }}$ to the desired $\bar{x}_{H_{2}}^{\text {set }}$ under the influence of a tubeside feed disturbance, additional temperature profiles are constructed based on the most essential criterion that the shapes (in the axial direction) of these additional temperature profiles have to match that of the original outer reforming tube wall temperature profile (owing to the overall shape and location of burner flames which does not change appreciably with time). The additional profiles are given by the following fourth-order polynomial,

$$
T_{\text {Wall }}(x)=\left[\begin{array}{lllll}
x^{4} & x^{3} x^{2} x^{1} x^{0}
\end{array}\right]\left[\begin{array}{c}
-0.0221 \\
0.8003 \\
-10.734 \\
64.416 \\
T_{\text {Wall }}^{\text {max }}-151.83
\end{array}\right]
$$

where $T_{\text {Wall }}^{\max }$ is the maximum outer reforming tube wall temperature of the temperature profile. Eq. 4 allows one to construct an entire outer reforming tube wall temperature profile along the reforming tube length based on its maximum temperature value and consequently affect the $\bar{x}_{H_{2}}^{\text {outlet }}$.

\subsection{Governing equations}

In the microscopic view of the reforming tube, the physico-chemical phenomena of transport and reaction processes that generate hydrogen fuel from steam 


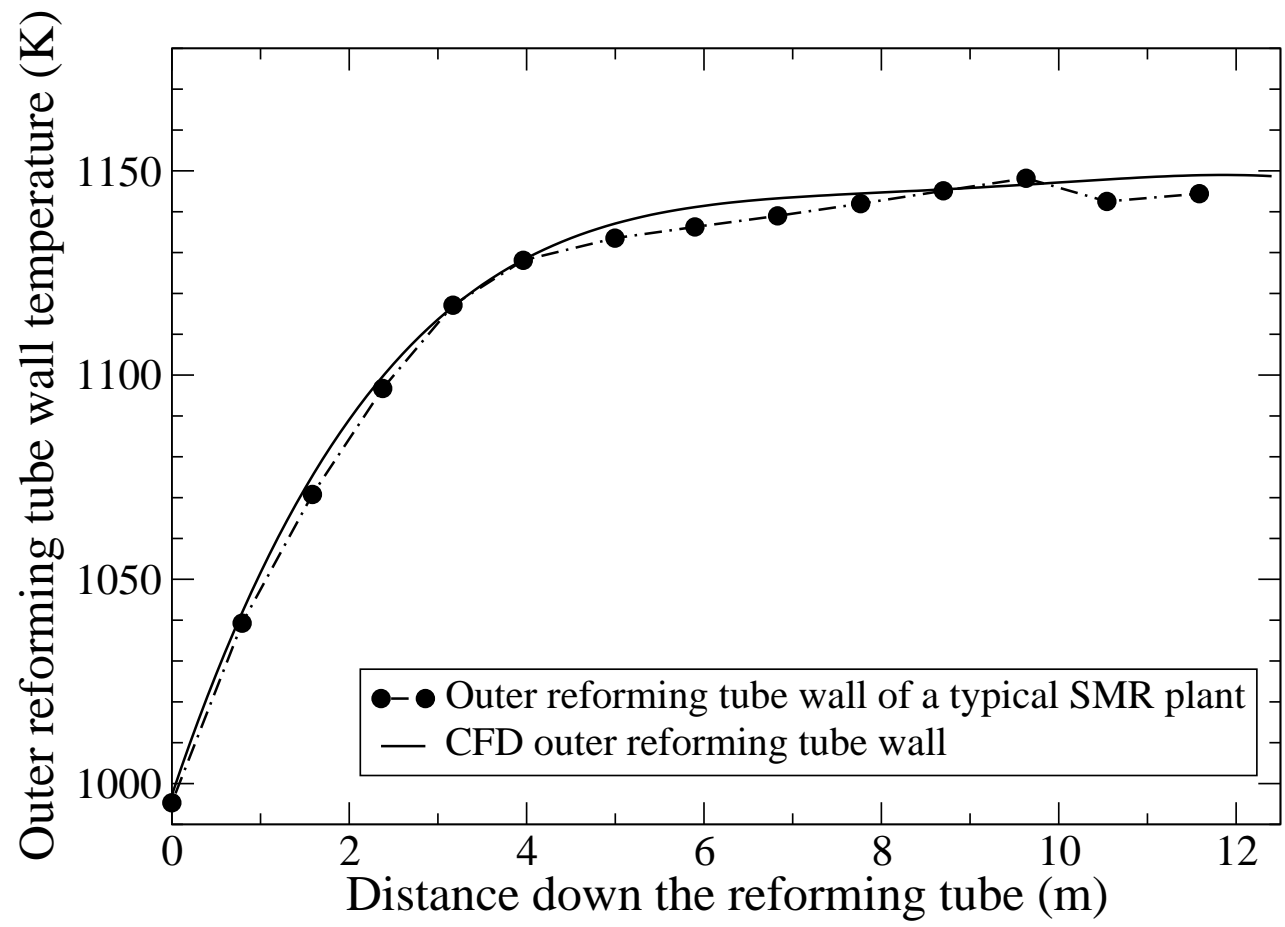

Figure 5: Outer reforming tube wall temperature versus distance down the reforming tube for the CFD simulations, which depicts the direct comparison between the fourth order polynomial temperature profile, i.e., Eq. 3 and that of publicly available SMR plant data Latham (2008).

Table 4: Reforming tube material properties.

\begin{tabular}{ll}
\hline Density, $\rho_{t}$ & $7720 \mathrm{~kg} / \mathrm{m}^{3}$ \\
\hline Heat Capacity, $C_{p, t}$ & $502 \mathrm{~J} /(\mathrm{kg} \cdot \mathrm{K})$ at $1144 \mathrm{~K}$ \\
\hline Thermal Conductivity, $k_{t}$ & $29.58 \mathrm{~W} /(\mathrm{m} \cdot \mathrm{K})$ at $1144 \mathrm{~K}$ \\
\hline Emissivity, $\epsilon_{t}$ & 0.85 at $1144 \mathrm{~K}$ \\
\hline
\end{tabular}

and methane are closely coupled and utterly complex. Initially, a convective mass transfer process driven primarily by the reactant concentration gradients between a flowing bulk gas mixture and an infinitesimally thin stationary layer around the catalytic surface takes place. Next, a molecular diffusion process mainly driven by 


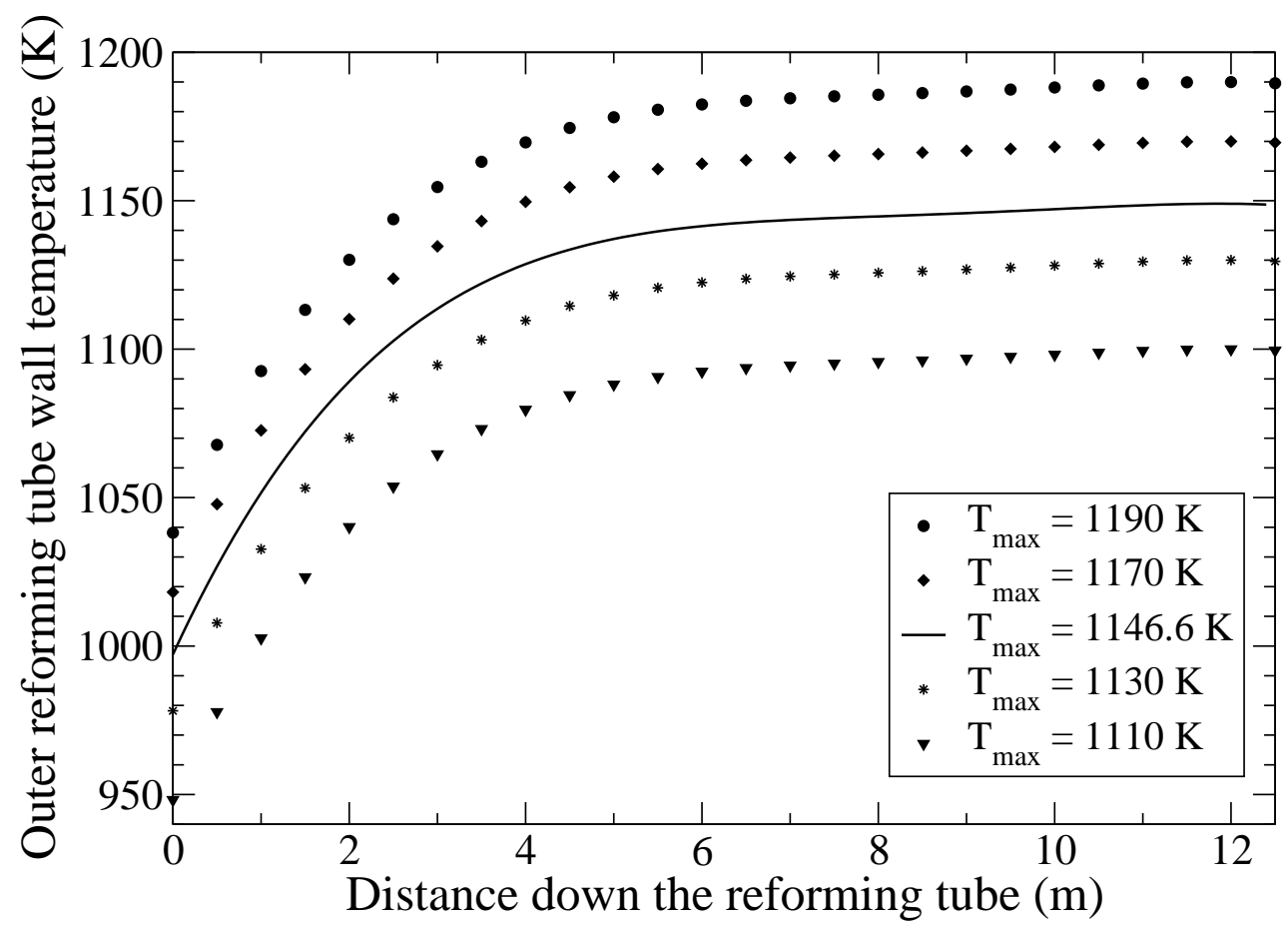

Figure 6: Outer reforming tube wall temperature versus distance down the reforming tube for the CFD simulations, which represents the calculated outer reforming tube wall temperature profiles generated by Eq. 4 and the fitted outer reforming tube wall temperature profile (solid line) based on the publicly available SMR plant data Latham (2008)

the reactant concentration and temperature gradients $(\mathrm{Eq} .6 \mathrm{~g})$ between an infinitesimally thin stationary layer around the catalyst surface and a catalyst medium allows the reactants to diffuse into the catalyst pores and then finally arrive at the catalyst active sites, where the endothermic SMR reactions take place. Upon the formation of the products, they immediately desorb from the catalyst active sites, diffuse back to the catalyst surface and eventually reenter the flowing bulk gas mixture. It is worth noting that the series of consecutive molecular-level elementary steps of the endothermic SMR reactions is still largely unknown. As a result, a kinetic model that provides a detailed treatment of these catalyst-specific 
phenomena would be unnecessarily complex from the point of view of CFD modeling; therefore, the kinetic model used in this paper is derived based on a widelyaccepted intrinsic SMR reaction kinetic model Xu and Froment (1989) to lessen the computational burden without sacrificing the accuracy of the simulation results (Sec. 2.3).

In the macroscopic view of the reforming tube, the significant axial pressure gradient across the reforming tube length and radial gradients near the reforming tube entrance are well-acknowledged. This, in turn, motivates us to develop two-dimensional (2D) governing equations that are capable of accounting for the presence of the catalyst network formed by the solidly packed catalyst particles. It is important to thoroughly understand the contribution of the catalyst network to the SMR reactions in order to develop an appropriate process model. Specifically, the catalyst network not only facilitates the formation of hydrogen fuel from the naturally stable and slowly-reacting materials, i.e., steam and methane, but also enhances the rate of convective thermal energy transfer from the heated outer reforming tube wall to the flowing bulk gas mixture by expanding its contact area. Additionally, due to the intrinsic resistance properties of the catalyst network, i.e., the inertial resistance and viscous resistance previously mentioned in Sec.2.5, the catalyst network acts as a physical obstacle that interferes with the flow of the tube-side gas mixture to generate turbulence. This obstacle enhances mixing efficiency to radially homogenize the moving fluid. Furthermore, under the influence of flow resistances induced by the catalyst network, the residence time of all species in the reforming tube is also increased, which allows the SMR reac- 
tions $\mathrm{Xu}$ and Froment (1989) to reach equilibrium prior to the process gas exiting the reforming tube. Based on the aforementioned considerations, the $2 \mathrm{D}$ governing equations, i.e., the continuity equation (Eq. 5) and the momentum (Eq. 6a), energy (Eq. 6b) and species material (Eq. 6c) balances, of the SMR process taking place inside the reforming tube are formulated as follows, so that the presence of the catalyst network is explicitly accounted for:

$$
\frac{\partial}{\partial t}\left(\gamma \rho_{\text {fluid }}\right)+\nabla \cdot\left(\gamma \rho_{\text {fluid }} \vec{v}\right)=0
$$




$$
\begin{aligned}
& \frac{\partial}{\partial t}\left(\gamma \rho_{\text {fluid }} \vec{v}\right)+\nabla \cdot\left(\gamma \rho_{\text {fluid }} \vec{v} \vec{v}\right)= \\
& \quad-\gamma \nabla P+\nabla \cdot(\gamma \vec{\tau})+\gamma \vec{B}_{f}-\left(\frac{\gamma^{2} \mu}{\alpha} \vec{v}+\frac{\gamma^{3} C_{2}}{2} \rho|\vec{v}| \vec{v}\right) \\
& \frac{\partial}{\partial t}\left(\gamma \rho_{\text {fluid }} E_{\text {fluid }}\right)+\frac{\partial}{\partial t}\left((1-\gamma) \rho_{\text {solid }} E_{\text {solid }}\right) \\
& +\nabla \cdot\left(\vec{v}\left(\rho_{\text {fluid }} E_{\text {fluid }}+P\right)\right)= \\
& \quad \nabla \cdot\left[k_{\text {eff }} \nabla T-\left(\sum_{i} h_{i} J_{i}\right)+\vec{\phi}\right]+\sum_{i} \Delta H_{\text {rxn }, i} \\
& \frac{\partial}{\partial t}\left(\gamma \rho_{\text {fluid }} Y_{i}\right)+\nabla \cdot\left(\gamma \rho_{\text {fluid }} \vec{v} Y_{i}\right)=-\nabla \cdot\left(\gamma \overrightarrow{J_{i}}\right)+R_{i}
\end{aligned}
$$

with

$$
\begin{aligned}
& \vec{v}_{\text {superficial }}=\gamma \vec{v} \\
& \vec{\tau}=\mu\left[\left(\nabla \vec{v}+\nabla \vec{v}^{T}\right)-\frac{2}{3} \nabla \cdot \vec{v} I\right] \\
& k_{\text {eff }}=\gamma k_{\text {fluid }}+(1-\gamma) k_{\text {solid }} \\
& \vec{J}_{i}=\left(\rho_{\text {fluid }} D_{m, i}+\frac{\mu_{t}}{S c_{t}}\right) \nabla Y_{i}-D_{T, i} \frac{\nabla T}{T} \\
& \vec{\phi}=2 \mu\left[\left(\frac{\partial v_{x}}{\partial x}\right)^{2}+\left(\frac{\partial v_{y}}{\partial y}\right)^{2}-\frac{1}{3}(\nabla \cdot \vec{v})^{2}\right] \\
& \quad+\mu\left[\frac{\partial v_{y}}{\partial x}+\frac{\partial v_{x}}{\partial y}\right]^{2}
\end{aligned}
$$

where $\mu, \mu_{t}, \vec{v}, \vec{v}_{\text {superficial }}, \rho_{\text {fluid }}$ and $\vec{B}_{f}$ are the molecular viscosity, turbulent viscosity, physical velocity vector, superficial velocity vector, average density, and body force of the bulk tube-side gas mixture respectively, $\gamma, \rho_{\text {solid }}$ and $\alpha$ are the porosity, density and absolute permeability of the catalyst network respectively, $\frac{1}{\alpha}$ and $C_{2}$ represent the viscous resistance coefficient and inertial resistance coef- 
ficient (previously introduced in Sec. 2.5), $E_{\text {fluid }}$ and $E_{\text {solid }}$ are the total energy of the tube-side gas mixture and of the catalyst network, $P$ is the static pressure, $\sum_{i} \Delta H_{r x n, i}$ is the total thermal energy generated by the SMR reactions, $k_{\text {fluid }}$, $k_{\text {solid }}$ and $k_{\text {eff }}$ are the thermal conductivities of the tube-side gas mixture, of the catalyst network and of the overall medium, $Y_{i}, D_{m, i}, D_{T, i}, \vec{J}_{i}$ and $R_{i}$ are the mass fraction, mass diffusion coefficient, thermal diffusion coefficient, turbulent mass diffusion flux and overall rate of chemical reaction of species $i, S c_{t}$ is the turbulent Schmidt number and is set to 0.7 by default, and $\vec{\tau}$ and $I$ are the stress tensor and unit tensor, respectively. It is important to note that the chemical rate equations are formulated to account for the effects of internal and external diffusion limitations on the observed rates as well as for the presence of the catalyst particles inside the reforming tube, and therefore, it would be unnecessary and incorrect to multiply $R_{i}$ in Eq. 6C and $\sum_{i} \Delta H_{r x n, i}$ in Eq. 6b by $\gamma$ in an attempt to account for the effects of the catalyst network. Additionally, detailed microscopic transport and chemical reaction processes are described by the $2 \mathrm{D}$ governing equations, i.e., $\left[\nabla \cdot\left(\sum_{i} h_{i} J_{i}\right)\right]$ in Eq. 6b represents the transport of enthalpy due to molecular diffusion, and $\left[\left(\nabla \vec{v}+\nabla \vec{v}^{T}\right)-\frac{2}{3} \nabla \cdot \vec{v} I\right]$ in Eq. 6e represents the effect of volume dilation.

Due to the high Reynolds number of the tube-side gas mixture, which is estimated to be $\sim 5500$ based on the inlet conditions of the tube-side feed as shown in Table 2, the semi-empirical standard two-equation turbulent kinetic energy and turbulent dissipation rate $(k-\epsilon)$ model Jones and Launder (1972); Launder and Sharma (1974) developed from the Reynolds-averaged Navier-Stokes 
(RANS) equations is employed to describe the complex turbulence phenomena inside the reforming tube. The $k-\epsilon$ turbulence model presented in Eq. 7 below is applicable for a wide range of flows and is relatively computationally inexpensive, though it still yields reasonably accurate estimates, and it is relatively simple to implement and easy to converge from the point of view of CFD simulation. This model has the form:

$$
\begin{aligned}
& \frac{\partial}{\partial t}\left(\gamma \rho_{\text {fluid }} k\right)+\nabla \cdot\left(\gamma \rho_{\text {fluid }} \vec{v} k\right)=\nabla \cdot\left[\gamma\left(\mu+\frac{\mu_{t}}{\sigma_{k}}\right) \nabla k\right] \\
& +\gamma G_{k}+\gamma G_{b}-\gamma \rho_{\text {fluid }} \epsilon-\gamma Y_{M} \\
& \frac{\partial}{\partial t}\left(\gamma \rho_{\text {fluid }} \epsilon\right)+\nabla \cdot\left(\gamma \rho_{\text {fluid }} \epsilon \vec{v}\right)=\nabla \cdot\left[\gamma\left(\mu+\frac{\mu_{t}}{\sigma_{\epsilon}}\right) \nabla \epsilon\right] \\
& +\gamma C_{1 \epsilon} \frac{\epsilon}{k}\left(G_{k}+C_{3 \epsilon} G_{b}\right)-\gamma C_{2 \epsilon} \rho \frac{\epsilon^{2}}{k} \\
& \left\{\begin{array}{l}
\mu_{t}=\rho_{\text {fluid }} C_{\mu} \frac{k^{2}}{\epsilon} \\
C_{3 \epsilon}=\tanh \left|\frac{v}{u}\right|^{2} \\
G_{b}=\beta g_{i} \frac{\mu_{t}}{P r_{t}} \frac{\partial T}{\partial x_{i}} \\
G_{k}=-\rho u_{i}^{\prime} u_{j}^{\prime} \frac{\partial u_{j}}{\partial x_{i}} \\
\beta=-\frac{1}{\rho}\left(\left.\frac{\partial \rho}{\partial T}\right|_{P}\right.
\end{array}\right.
\end{aligned}
$$

where $k$ and $\epsilon$ are the turbulent kinetic energy and turbulent dissipation rate respectively, $G_{k}$ and $G_{b}$ are the generation of turbulent kinetic energy due to the mean velocity gradients and buoyancy respectively, $Y_{M}$ is the contribution of the fluctuating dilation in compressible turbulence to the overall dissipation rate, $\sigma_{\epsilon}=1.0$ and $\sigma_{k}=1.3$ are the turbulent Prandtl numbers for $\epsilon$ and $k$ respectively, 
$C_{1 \epsilon}=1.44, C_{2 \epsilon}=1.92$ and $C_{\mu}=0.09$ are default constants of the standard $k-\epsilon$ model, $P r_{t}=0.85$ is the turbulent Prandtl number for energy, $g_{i}$ is the component of the gravitational vector in the ith direction, $\beta$ is the thermal expansion coefficient, and $v$ and $u$ are the components of the flow velocity parallel and perpendicular, respectively, to the gravitational vector. It is worth noting that all default values of the aforementioned constants are determined empirically by experiments for fundamental turbulent flows, i.e., boundary layers and mixing layers, and have been verified to be suitable for a wide range of wall-bounded and free shear flow applications Inc. (Nov. 2013a). Furthermore, turbulent flows are significantly affected by the walls. The accuracy of the near-wall modeling decides the fidelity of numerical solutions. Specifically, it is in the near-wall region that the solution variables have large gradients, i.e., the momentum, material and energy fluxes have large magnitudes. Therefore, an accurate representation of the flow in the near-wall region is required for successful predictions of wall-bounded turbulent flows. For this single reforming tube CFD model, the enhanced wall treatment function in Fluent is applied as the near wall treatment method. The enhanced wall treatment $\epsilon$-Equation is suitable for certain fluid dynamics especially for those with lightly turbulent flow, and it only requires a few nodes in the boundary layer when using the $k-\epsilon \operatorname{model}$ Inc. (Nov. 2013a).

\subsection{Simulation Results}

\subsubsection{CFD Model of Single Reforming Tube: Results Comparison}

Using a parallel computational environment with message passing interface technology, the simulation of the single reforming tube CFD model converges in 
about 5 minutes with the steady solver in a 4-core CPU desktop computer. The steady-state results of the simulations are displayed in Figs. 7. 8 , We note here that due to the large length to diameter ratio of the reforming tube, in all plots of the simulation results, the radius is scaled up by 20 times which is convenient and is done for display purposes only. It is worth noting that the pressure profile in this simulation is radially uniform, which is the result of the direction definitions of the resistance coefficients in Section 2.5. In addition, based on the wall temperature profile in Fig. 5, the corresponding inner reforming tube wall temperature profile at the steady-state from the converged model is displayed in Fig. 9, and the corresponding steady-state heat flux profile through the tube wall is shown in Fig. 10 . 


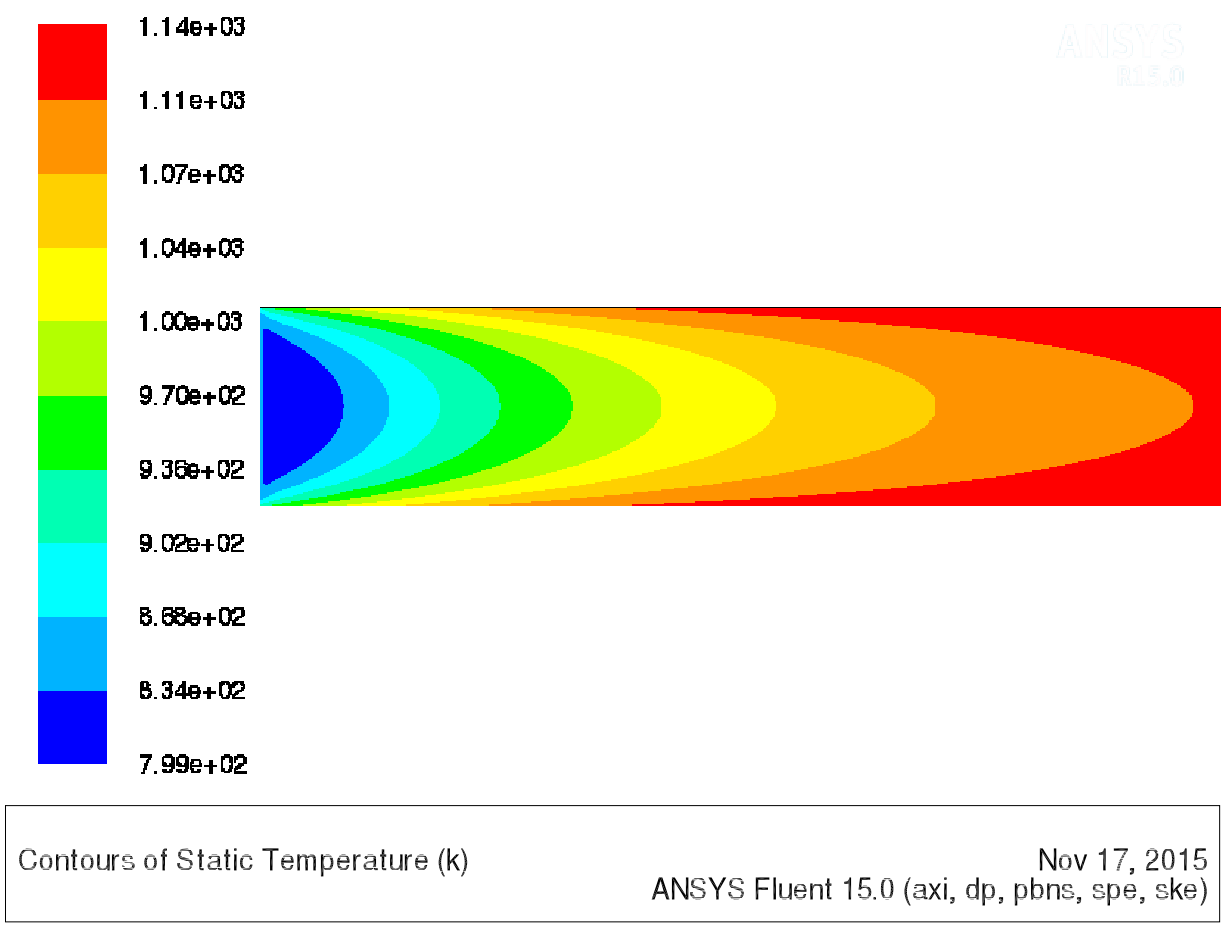

Figure 7: Temperature profile from the reforming tube CFD simulation, where the outer reforming tube wall temperature profile (Fig. 5) is that fitted based on the available SMR plant data Latham (2008).

Reforming tube outlet data (not reported here for proprietary reasons) from a typical hydrogen plant with the same tube-side inlet conditions, the same tube geometric structure and similar catalyst and tube material properties as described in Secs. 2.1, 2.4 and 2.5, and also the same wall temperature profile as that reported in Fig. 5, is used to validate the CFD single tube model. The validation is performed by comparing the data of the process gas at the reforming tube outlet (Table 5) from the CFD simulation to that of the available proprietary industrial plant. All mole fraction values in Table 5 are area-weighted average values at the tube outlet. We found that our simulation results reported in Table 5 are very close 


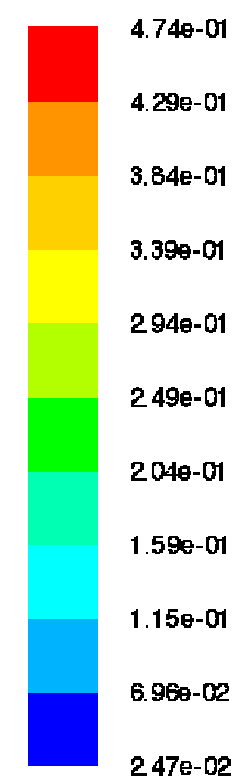

Contours of Mole fraction of h2

ANSYS Fluent 15.0 (axi, dp, pbns, spe, ske)

Figure 8: Hydrogen mole fraction profiles from the reforming tube CFD simulation, where the outer reforming tube wall temperature profile (Fig. 5) is that fitted based on the available SMR plant data Latham (2008).

to the plant data for temperature and species compositions.

Additionally, the average heat flux across the reforming tube wall produced by the CFD model is within the range of $45-90 \mathrm{~kW} / \mathrm{m}^{2}$ Rostrup-Nielsen (1984), a typical range reported in industry, and closely resembles that reported throughout literature. Namely, Dybkjaer reports the average value of $78.5 \mathrm{~kW} / \mathrm{m}^{2}$ Dvbkiaer (1995) and Froment and Bischoff report the average value of $75.6 \mathrm{~kW} / \mathrm{m}^{2}$ Froment and Bischoff (1990). Moreover, the work of Pantoleontos et al. on modeling and optimization of a reforming reactor also restricts the average heat flux to be less than $80.0 \mathrm{~kW} / \mathrm{m}^{2}$ Pantoleontos et al. (2012). Furthermore, the estimated average heat 


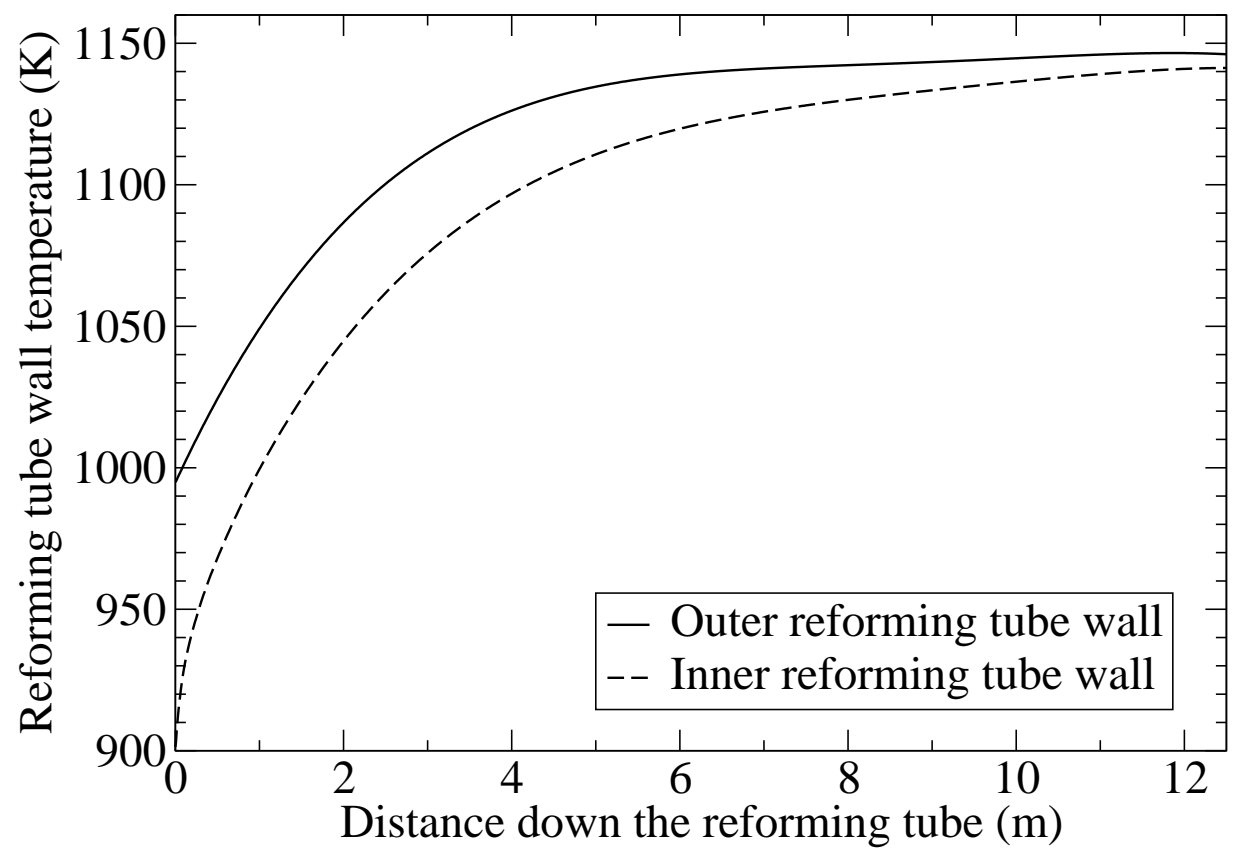
ture profiles, thermal conductivity of $106500 \frac{\mathrm{J}}{\mathrm{m} \cdot h \cdot K}$ and thickness of the reforming tube wall reported by Latham validates the accuracy of the CFD model Latham (2008).

Figure 9: Inner (dashed line) and outer (solid line) wall temperature profiles of the reforming tube.

flux of $65.6 \mathrm{~kW} / \mathrm{m}^{2}$ based on the outer and inner reforming tube wall tempera-

Remark 1. The outlet tube-side mixture compositions are at their equilibrium state at the exit of the reactor which is determined by the outlet tube-side mixture temperature. Further, the average hydrogen mole fraction in the tube-side mixture measured at the tube outlet, $\bar{x}_{H_{2}}^{\text {outlet }}$, can be mathematically expressed as a function of the maximum reforming tube wall temperature, $T_{W a l l}^{\max }$. It is important to 


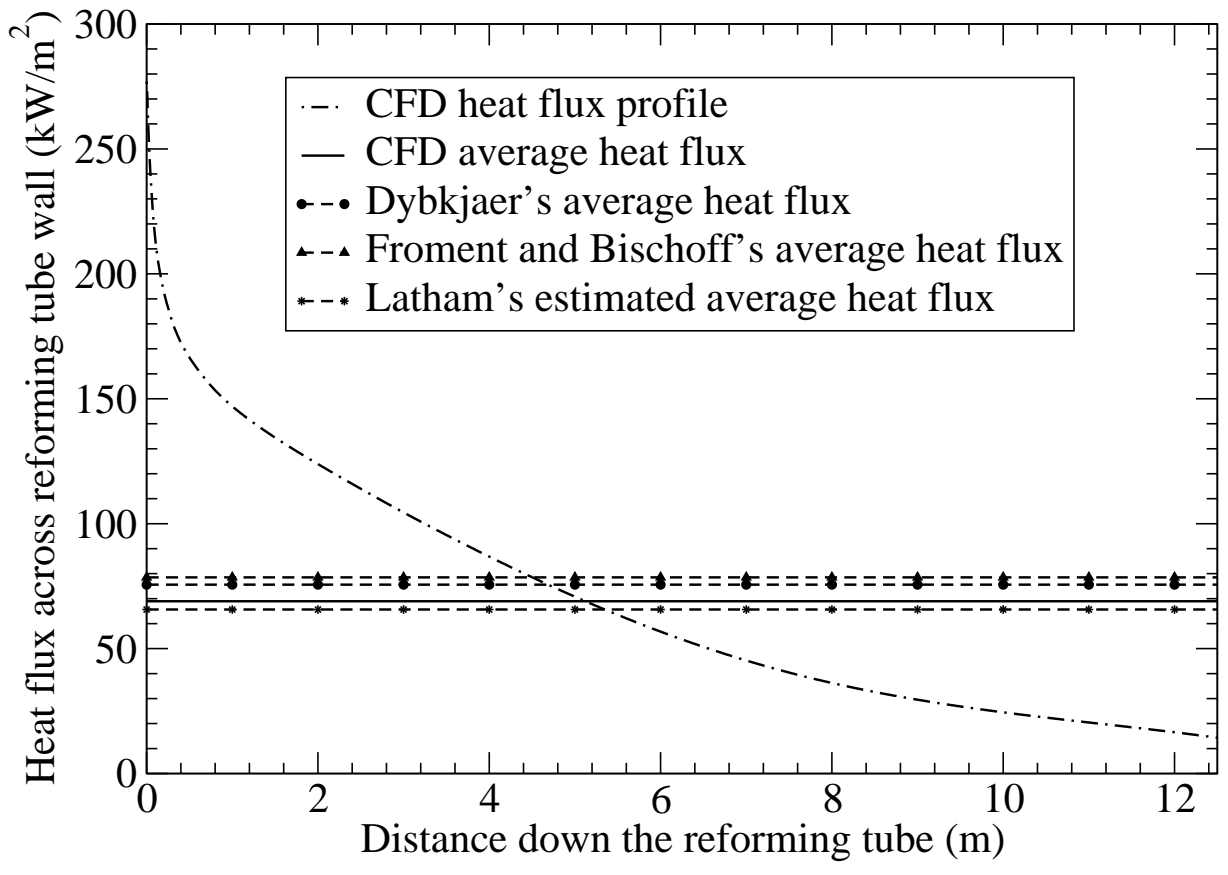

Figure 10: Heat flux profile across the reforming tube wall.

note that $T_{W \text { all }}^{\max }$ is not necessarily the temperature of the tube-side mixture at the outlet, which depends not only on $T_{\text {Wall }}^{\max }$, but also depends on the thermal conductivity of the reforming tube wall, the effective heat transfer coefficient between the tube-side mixture and the catalyst network, and especially, the rate of energy consumption by the endothermic SMR reactions. A major benefit of creating a CFD model of the industrial-scale reforming tube is that it allows relationships between important quantities within the reformer to be determined for control or other analysis purposes. For example, it allows the generation of the collection of data at constant tube-side feed conditions needed to construct the relationship between $\bar{x}_{H_{2}}^{\text {outlet }}$ and $T_{\text {Wall }}^{\max }$ used for control purposes later in this work. Additionally, the industrial-scale reforming tube CFD model creates more opportunities for 
future investigations. Specifically, the CFD model allows us to access additional information about the tube-side mixture at the reforming tube outlet, e.g., the areaweighted average temperature of the tube-side mixture denoted by $\bar{T}_{\text {Mixture }}^{\text {outlet }}$, which can be used to construct the relationship between $\bar{T}_{\text {Mixture }}^{\text {outlet }}$ and $T_{\text {Wall }}^{\max }$ to construct, for example, a Gibbs reactor model.

Additional effort to validate the simulation result generated by the single reforming tube CFD model in order to declare with high certainty that the CFD model can replace expensive traditional on-site parametric study, and to also demonstrate that the tube-side gas mixture at the reforming tube outlet is at equilibrium conditions is conducted. Specifically, a Gibbs reactor model of a steady state chemical and petroleum process simulation software known as Pro//II is adopted to simulate the reforming tube to determine the species composition at chemical equilibrium by solving the energy and material conservation equations based on minimizing the Gibbs free energy of all chemical species involved in this process. Furthermore, the greatest advantage of the Gibbs reactor model is that it does not require the knowledge of chemical process in the reforming tube including detailed reaction mechanism or kinetic model, which in turn validate the global reaction mechanism and kinetic models of SMR process we implemented in the single reforming tube CFD model. In this effort, the Gibbs reactor model, which requires only two input parameters, i.e a Gibbs reactor feed stream, which is identical to that of tube-side feed (shown in Table 2), and a Gibbs reactor duty, which is set to the total thermal energy absorbed by the reforming tube of $341.045 \mathrm{~kW}$, is simulated, and the corresponding simulation results are shown in 
Table 5. The simulation results generated by the Gibbs reactor model are in close agreement with that generated by the single reforming tube CFD model, which in turn support the accuracy of the CFD model, validate our choice of the global reaction mechanism and kinetic model of SMR process, and also demonstrate that the tube-side gas mixture at the reforming tube outlet is at equilibrium conditions.

Table 5: Single reforming tube results.

\begin{tabular}{lllc}
\hline & CFD Result & Pro/II & $\begin{array}{l}\text { \% Difference with } \\
\text { respect to CFD result }\end{array}$ \\
\hline$\Delta P(k P a)$ & 212.83 & N/A & N/A \\
\hline$P_{\text {outlet }}(k P a)$ & 3044.0 & 3044.0 & 0.00 \\
\hline $\bar{x}_{H_{2}}^{\text {outlet }}$ & 0.4645 & 0.4650 & 0.11 \\
\hline $\bar{x}_{H_{2} O}^{\text {outlet }}$ & 0.3467 & 0.3452 & 0.43 \\
\hline $\bar{x}_{C H_{4}}^{\text {outlet }}$ & 0.0426 & 0.0422 & 0.94 \\
\hline $\bar{x}_{C O}^{\text {outlet }}$ & 0.0873 & 0.0869 & 0.46 \\
\hline $\bar{x}_{C O_{2}}^{\text {outlet }}$ & 0.0588 & 0.0607 & 3.23 \\
\hline $\begin{array}{l}\text { Average heat } \\
\text { flux }\left(\frac{\mathrm{kW}}{\mathrm{m}^{2}}\right)\end{array}$ & 68.972 & $\mathrm{~N} / \mathrm{A}$ & $\mathrm{N} / \mathrm{A}$ \\
\hline $\begin{array}{l}\text { Total absorbed } \\
\text { heat }(\mathrm{kW})\end{array}$ & 341.045 & 341.055 & $\sim 0.00$ \\
\hline
\end{tabular}

\section{Feedback control design and closed-loop simulation results}

\subsection{Open-loop dynamics}

We are primarily interested in the regulation of $\bar{x}_{H_{2}}^{\text {outlet }}$ when the reforming tube is subjected to tube-side feed disturbances, where the control objective is to maintain the current $\bar{x}_{H_{2}}^{\text {set }}$, or when changes in industrial plant objectives occur, where the control objective is to attain a new $\bar{x}_{H_{2}}^{\text {set }}$. 
Among all simulation settings described in Section 2 , the outer reforming tube wall temperature profile is chosen as the single manipulated input due to the endothermic nature of the SMR reactions. As a result, $\bar{x}_{H_{2}}^{\text {outlet }}$ can be manipulated by adjusting the outer reforming tube wall temperature profile, which depends on the fuel flow rates to the burners around the reforming tube. It should be noted that the maximum value of an outer reforming tube wall temperature profile $\left(T_{\text {Wall }}^{\max }\right)$ can be used in Eq. 4 to construct an entire profile along the reforming tube, and therefore, a specific $T_{\text {Wall }}^{\max }$ can represent a unique outer reforming tube wall temperature profile. The temperature dependence of $\bar{x}_{H_{2}}^{\text {outtet }}$ is demonstrated in Fig. 12 for a wide range of outer reforming tube wall temperature profiles for which $T_{\text {Wall }}^{\max }$ varies from $1100 K$ to $1200 K$. Due to the endothermic nature of the SMR reactions, higher values of $\bar{x}_{H_{2}}^{\text {outlet }}$ are observed for temperature profiles with higher $T_{\text {Wall }}^{\max }$.

In this work, all CFD reforming tube simulations under feedback control and under open-loop control are carried out in a parallel computational environment with message passing interface technology with a built-in, explicit-time-stepping, transient solver. Transient simulation may reveal dynamics of a system that cannot be obtained under steady-state simulation Pantoleontos et al. (2012). In particular, Barton reported that the catalyst core temperatures of the water-gas shift reactors in an IGCC-TIGAS polygeneration plant can exceed the maximum steady-state value by $100 \mathrm{~K}$ during start-up, which would not be detected under steady-state simulation Adams and Barton (2009). Additionally, the transient solver can be used to determine the steady-state solution when a steady-state model is highly 


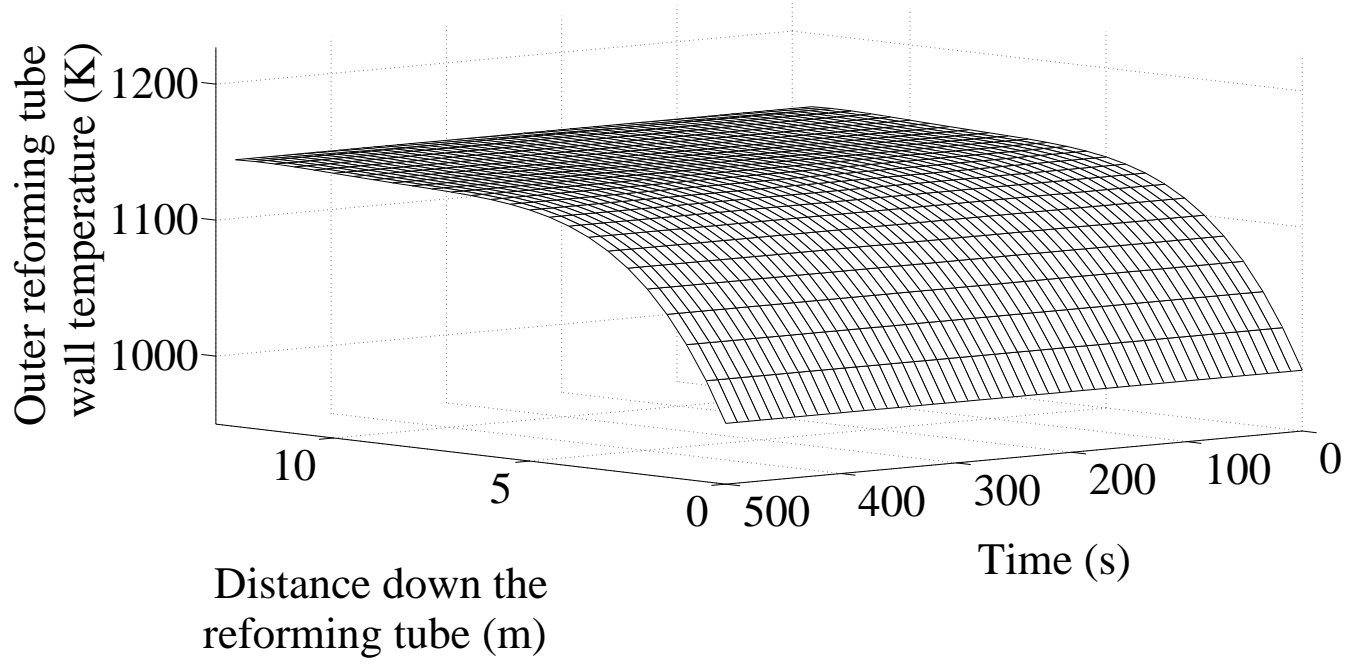

Figure 11: The pre-determined outer reforming tube wall temperature trajectory with time for open-loop control with $\bar{x}_{\mathrm{H}_{2}}^{\text {set }}=0.465$.

unstable, e.g., natural convection problems with Rayleigh number in the transition regime Inc. (Nov. 2013a). CFD simulations under open-loop control using a constant pre-determined outer wall temperature trajectory (e.g., Fig. 11) as the process manipulated input and built with fixed simulation settings can successfully drive $\bar{x}_{H_{2}}^{\text {outlet }}$ to the desired $\bar{x}_{H_{2}}^{\text {set }}$ under a strictly disturbance-free environment as shown in Fig. 13, However, due to the absence of the feedback mechanism, open-loop control is unable to recognize or handle the tube-side feed disturbances, which results in a substantial deviation of the steady-state $\bar{x}_{H_{2}}^{\text {outlet }}$ from the desired set-point, $\bar{x}_{\mathrm{H}_{2}}^{\text {set }}$. The aforementioned robustness issue motivates us to design feedback control schemes including proportional (P), proportional-integral (PI) and optimization-based control schemes, which are utilized to produce a feedbackbased manipulated input in order to drive $\bar{x}_{H_{2}}^{\text {outlet }}$ to the desired $\bar{x}_{H_{2}}^{\text {set }}$ in the presence 


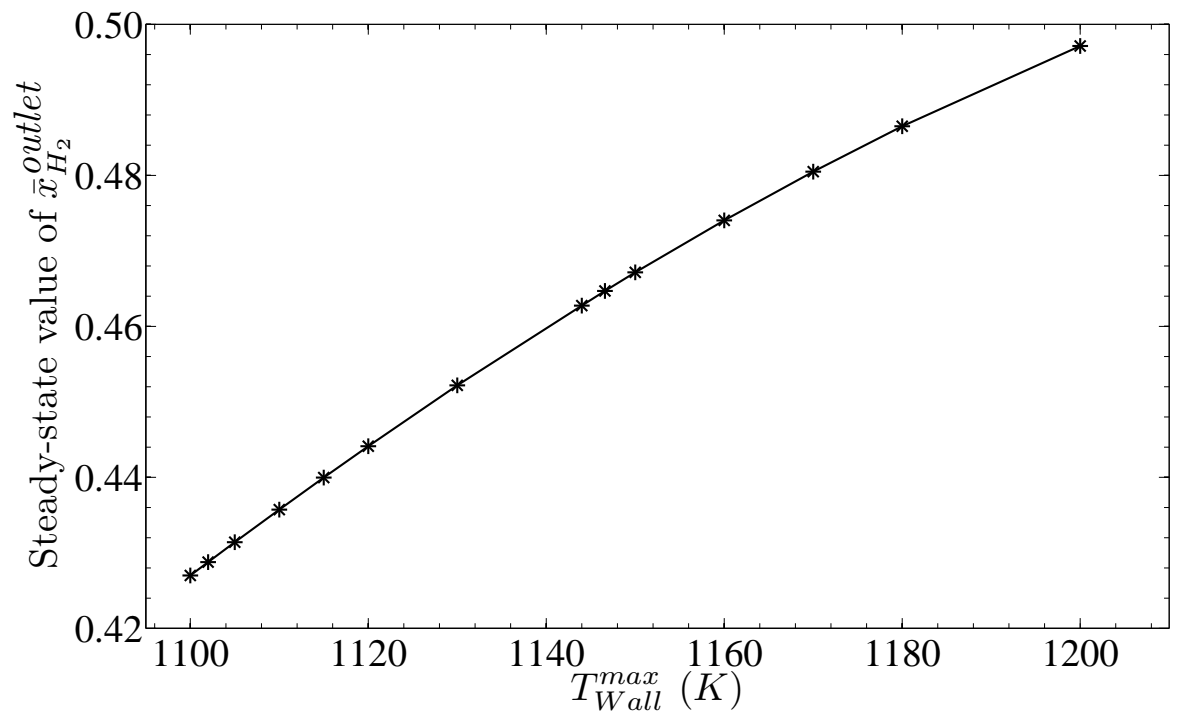

Figure 12: The expected steady-state value of $\bar{x}_{\mathrm{H}_{2}}^{\text {outlet }}$ at different constant outer reforming tube temperature profiles which are presented by their corresponding $T_{W a l l}^{\max }$. The relationship between $\bar{x}_{H_{2}}^{\text {outlet }}$ and $T_{\text {Wall }}^{\text {max }}$ is captured by the second-order polynomial, $T_{\text {Wall }}^{\text {max }}=5648.6\left(\bar{x}_{H_{2}}^{\text {outlet }}\right)^{2}-$ $3814.7 \bar{x}_{\mathrm{H}_{2}}^{\text {outlet }}+1699.5$, which is used to generate an appropriate manipulated input for the openloop CFD simulations based on the desired $\bar{x}_{H_{2}}^{\text {set }}$ (Fig. 111).

of tube-side feed disturbances.

\subsection{Feedback controller design}

In this work, the primary objectives of the feedback control schemes are to drive $\bar{x}_{H_{2}}^{\text {outlet }}$ to the desired $\bar{x}_{H_{2}}^{\text {set }}$ in the presence of the tube-side feed disturbance, and to speed up the dynamics of the process to allow the system to quickly advance to the optimized steady-state operating condition. All simulation settings of the closed-loop simulations are the same as those used in the previously studied open-loop system. It is important to emphasize that the disturbance and set-point changes are added to the process after it has already achieved a steady-state under a constant wall temperature profile. 


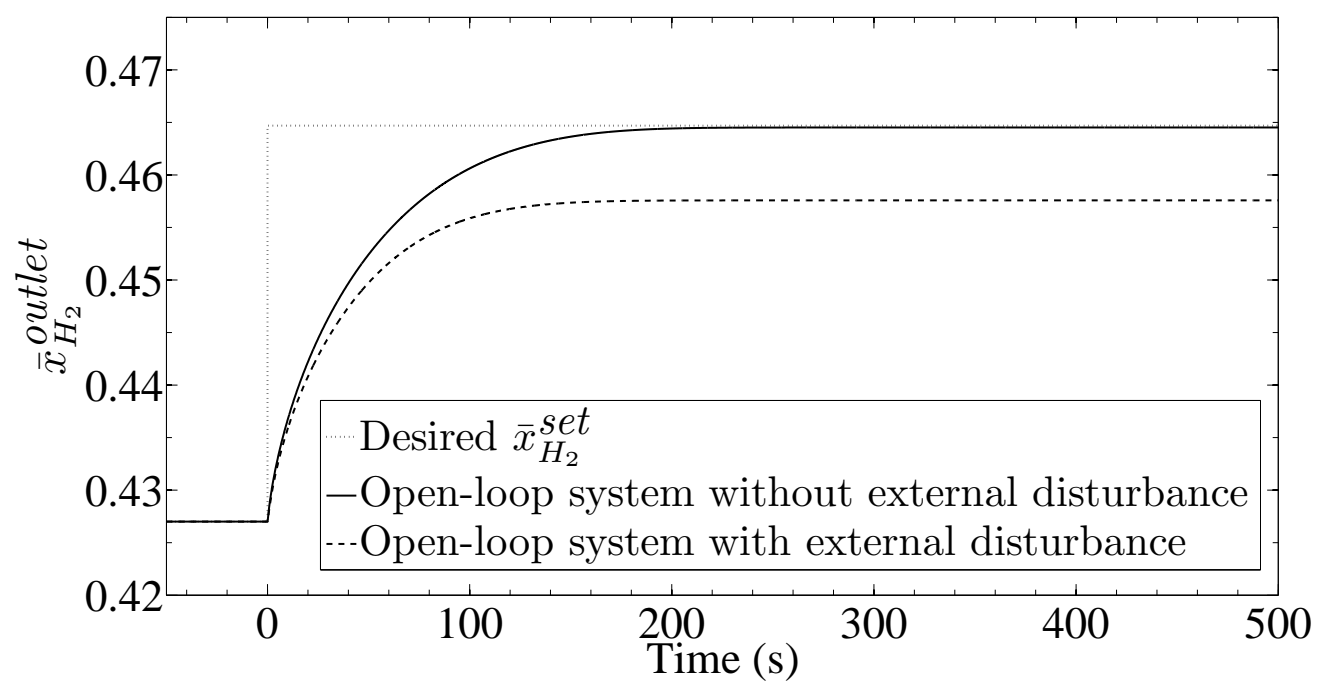

Figure 13: The propagation of $\bar{x}_{H_{2}}^{\text {outlet }}$ with time under open-loop control with $\bar{x}_{H_{2}}^{\text {set }}=0.465$, when the tube-side mass flow rate is kept at its nominal value (solid line), and when it is increased by $20 \%$ (dashed line). Fig. 13 demonstrates that open-loop control is not robust and cannot be used to maintain $\bar{x}_{H_{2}}^{\text {set }}$ at 0.465 when a change in the operating condition is imposed.

These feedback control schemes make the control objectives feasible and provide robustness against the tube-side feed disturbance, and thus resolve the critical drawback of open-loop control. It is desired that under each feedback control scheme, the closed-loop system is initially operated in a high temperature regime favoring the formation of hydrogen fuel resulting in a fast increase of $\bar{x}_{H_{2}}^{\text {outtet }}$ toward the desired $\bar{x}_{H_{2}}^{\text {set }}$ due to the endothermic nature of the SMR reactions described in Eq. 1. After the initial burst of hydrogen fuel, it is desired that the closed-loop system is then kept in a moderately high temperature regime, which effectively slows down the rapid formation of hydrogen fuel to prevent unnecessary aggressive control action. To design feedback control schemes to accomplish the operating strategy, a data-driven modeling technique is used to obtain a process transfer 
function describing the evolution of $\bar{x}_{H_{2}}^{\text {outlet }}$ based on the CFD simulation result for a step-change of the maximum outer reforming tube wall temperature from $T_{\text {Wall }}^{\max }(0)=1100 \mathrm{~K}$ to $T_{W \text { all }}^{\max }(t)=1110 \mathrm{~K}$ (which is discussed in greater detail below). Then, this approximate model capturing the dominant dynamics of the reforming tube is combined with classical feedback control to form a closed-loop system.

It is worth noting that the process transfer function derived based on the response of $\bar{x}_{H_{2}}^{\text {outlet }}$ to a step-change of $T_{W \text { all }}^{\max }$ from $T_{\text {Wall }}^{\max }=1100 \mathrm{~K}$ to $T_{W \text { all }}^{\max }=$ $1110 K$ was compared with other transfer functions derived based on different step-changes of $T_{W a l l}^{\max }$. Specifically, different step changes in the input, i.e., step changes in $T_{\text {Wall }}^{\max }$ from $1110 \mathrm{~K}$ to $1130 \mathrm{~K}$, from $1130 \mathrm{~K}$ to $1160 \mathrm{~K}$ and from $1160 K$ to $1200 K$, were considered. The variations between the transfer functions (normalized) derived from the different sets of simulation results are negligible.

The closed-loop system is utilized in a parametric study to determine the appropriate control parameters, i.e., $K_{c}$ of $\mathrm{P}$ control or $K_{c}$ and $\tau_{I}$ of PI control. Specifically, the initial values of the P/PI parameters are obtained by the CohenCoon tuning method based on the response of the process output, $\bar{x}_{H_{2}}^{\text {outlet }}$, to a step-change of the process input chosen as the maximum outer reforming tube wall temperature. Then the process model derived from the data-driven modeling technique based on the aforementioned simulation result is used to build closedloop models with feedback control schemes to improve the controller parameter estimates. Finally, the P/PI parameters are incorporated into the closed-loop CFD models, and adjusted until the desired closed-loop responses are observed. 
Furthermore, the maximum allowable temperature value of $1200 \mathrm{~K}$ is taken into account in the control schemes to avoid the rupture of the reforming tube which can occur at exceedingly high operating temperatures. Additionally, this upper limit on the outer reforming tube wall temperature prevents the deposition of carbon on the reforming tube wall and on the surfaces of catalyst particles, which would significantly decrease the heat transfer rate to the flowing gas mixture and prevent the reactants from entering the catalyst active sites resulting in a lower $\bar{x}_{\mathrm{H}_{2}}^{\text {outlet }}$. Next, the minimum allowable temperature value of $987 \mathrm{~K}$ at the tube inlet is considered in the formulation of the control schemes to ensure that sufficient heat is transferred to the flowing tube-side gas mixture to facilitate the highly endothermic SMR reactions. Moreover, since the scope of our current work focuses on CFD modeling and controller design of the reforming tube instead of the SMR furnace, we will disregard the dynamics of the outer reforming tube wall temperature. Hence, we suppose that the outer reforming tube wall temperature can reach the predicted profile instantaneously. Lastly, we assume that measurements of $\bar{x}_{H_{2}}^{\text {outtet }}$ are available at all sampling instances. Classical P and PI control

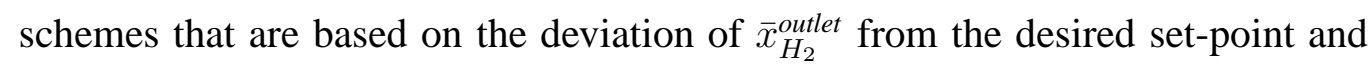
account for input constraints are presented as follows: 
P control scheme

$$
\left\{\begin{array}{l}
e\left(t_{k}\right)=\bar{x}_{H_{2}}^{\text {set }}-\bar{x}_{H_{2}}^{\text {outlet }}\left(t_{k}\right) \\
u_{P}\left(t_{k}\right)=K_{c}^{\prime} e\left(t_{k}\right) \\
T_{W a l l}^{\max }\left(t_{k}+\Delta t\right)= \\
\quad T_{\text {Wall }}^{\text {max }}(t=0)+u_{P}\left(t_{k}\right)
\end{array}\right.
$$

PI control scheme

$$
\begin{aligned}
& \left\{\begin{array}{l}
e\left(t_{k}\right)=\bar{x}_{H_{2}}^{\text {set }}-\bar{x}_{H_{2}}^{\text {outlet }}\left(t_{k}\right) \\
u_{P I}\left(t_{k}\right)=K_{c}\left(e\left(t_{k}\right)+\frac{1}{\tau_{I}} \int_{t_{0}}^{t_{k}} e(\tau) d \tau\right) \\
T_{\text {Wall }}^{\text {max }}\left(t_{k}+\Delta t\right)= \\
T_{\text {Wall }}^{\text {max }}(t=0)+u_{P I}\left(t_{k}\right)
\end{array}\right. \\
& T_{\text {Wall }}\left(x, t_{k}+\Delta t\right) \leq T_{\text {max }} \\
& T_{\text {Wall }}\left(x=0, t_{k}+\Delta t\right) \geq T_{\text {min }} \\
& \left.T_{\text {Wall }}\left(x, t_{k}+\Delta t\right)=\begin{array}{c}
-0.0221 \\
0.8003 \\
-10.734 \\
64.416
\end{array}\right] \\
& {\left[\begin{array}{c}
x^{4} x^{3} x^{2} x^{1} x^{0} \\
T_{W \text { all }}^{\text {max }}\left(t_{k}+\Delta t\right)-151.83
\end{array}\right]}
\end{aligned}
$$

where $t_{k}, t_{0}$ and $\Delta t=0.04$ seconds are the current time, the initial time, and the sampling time interval of the CFD simulation respectively, $e\left(t_{k}\right)$ represents the current deviation of $\bar{x}_{H_{2}}^{\text {outlet }}$ from the desired set-point $\bar{x}_{H_{2}}^{\text {set }}, T_{\min }$ and $T_{\max }$ are the minimum and maximum allowable reforming tube wall temperatures respectively, $K_{c}^{\prime}=1856.3$ and $u_{P}\left(t_{k}\right)$ are the controller gain and current controller output of the $\mathrm{P}$ control scheme, $K_{c}=1856.3, \tau_{I}=46.4$ and $u_{P I}\left(t_{k}\right)$ are the controller gain, 
controller time constant and current controller output of the PI control scheme, $T_{\text {Wall }}^{\max }\left(t_{k}+\Delta t\right)$ and $T_{\text {Wall }}^{\max }(t=0)$ are the predicted (i.e., prior to being saturated with the temperature constraints) and initial maximum outer reforming tube wall temperatures, respectively, and $T_{W a l l}\left(x, t_{k}+\Delta t\right)$ is the predicted outer reforming tube wall temperature profile. At the end of each sampling time interval, the

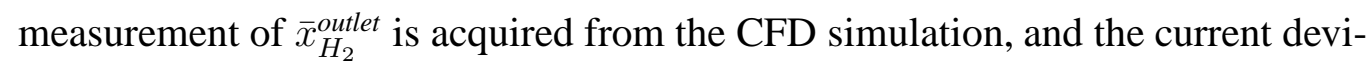
ation from the desired $\bar{x}_{H_{2}}^{\text {set }}\left(e\left(t_{k}\right)\right)$ is computed. Then, the control output $\left(u_{P I}\left(t_{k}\right)\right.$ or $\left.u_{P}\left(t_{k}\right)\right)$ is evaluated depending on the implemented control scheme, which allows $T_{\text {Wall }}^{\max }\left(t_{k}+\Delta t\right)$ to be estimated. The value of $T_{\text {Wall }}^{\max }\left(t_{k}+\Delta t\right)$ is subjected to Eq. 8c, if the constraint is not satisfied, $T_{W a l l}^{\max }\left(t_{k}+\Delta t\right)$ is set to the value of $T_{\max }$. Then, $T_{\text {Wall }}^{\max }\left(t_{k}+\Delta t\right)$ is used to compute the predicted wall temperature profile $\left(T_{W a l l}\left(x, t_{k}+\Delta t\right)\right)$, which is applied until the next sampling time, when the new value of $\bar{x}_{\mathrm{H}_{2}}^{\text {outlet }}$ is obtained from the CFD simulation.

\subsection{Closed-loop simulation results}

In this section, we investigate the closed-loop performance of the P and PI controllers, which adjust the manipulated input variable chosen to be the outer reforming tube wall temperature, to drive $\bar{x}_{H_{2}}^{\text {outlet }}$ to the desired $\bar{x}_{H_{2}}^{\text {set }}$ in the absence or presence of a tube-side feed disturbance. At the end of each sampling time interval, the measurement of $\bar{x}_{H_{2}}^{\text {outlet }}$ is acquired from the CFD simulation, and the control action, which is formulated following the scheme described in Eq. 8, is evaluated. Next, the control action is applied to the closed-loop system until the end of the next sampling time, when the new $\bar{x}_{H_{2}}^{\text {outlet }}$ is received from the CFD simulation. In our work, the performance of the feedback control schemes is 
evaluated based on two criteria: the improvement in the process dynamics and the final deviation of $\bar{x}_{H_{2}}^{\text {outlet }}$ from the desired $\bar{x}_{H_{2}}^{\text {set }}$. It is important to note that the process dynamics in this work is defined as the time duration that is needed for the $\bar{x}_{H_{2}}^{\text {outlet }}$ of the closed-loop system to first attain $99.99 \%$ of the desired $\bar{x}_{H_{2}}^{\text {set }}$. Unfortunately, due to the well-known drawback of P control, an offset of the final value of $\bar{x}_{H_{2}}^{\text {outlet }}$ is expected in the system under $\mathrm{P}$ control, and hence, the process dynamics cannot be evaluated based on the arrival of $\bar{x}_{\mathrm{H}_{2}}^{\text {outlet }}$ at the desired $\bar{x}_{\mathrm{H}_{2}}^{\text {set }}$. In the case of the $\mathrm{P}$ control scheme, the process dynamics is the time that is required for the closed-loop system to settle at its new steady-state.

In the case of set-point tracking control under a disturbance-free environment, the outer reforming tube wall temperature trajectory propagates differently under P control and PI control than under open-loop control (Fig. 11, Fig. 14(a) and Fig 14(b) . The open-loop control strategy adopts the outer reforming tube wall temperature profile based on the relationship between $\bar{x}_{H_{2}}^{\text {outlet }}$ and $T_{W a l l}^{\max }$ as shown in Fig. 12 with $\bar{x}_{H_{2}}^{\text {outlet }}=\bar{x}_{H_{2}}^{\text {set }}=0.465$. Because of the aforementioned closedloop operating policy described in Section 3.2, the process dynamics is greatly enhanced in the closed-loop system shown in Fig. 15. In particular, it takes $\sim 244$ seconds for $\bar{x}_{H_{2}}^{\text {outlet }}$ to attain its final value under open-loop control, while it takes $\sim 90$ seconds corresponding to an improvement of $63.1 \%$ under P control or $\sim 154$ seconds corresponding to an improvement of $36.9 \%$ under PI control, respectively. Nevertheless, the closed-loop system implemented with P control is unable to drive $\bar{x}_{\mathrm{H}_{2}}^{\text {outlet }}$ completely to the desired $\bar{x}_{\mathrm{H}_{2}}^{\text {set }}$, and the offset is estimated to be $3.18 \%$. 
Next, we turn our attention to the case of set-point tracking control under a tube-side feed disturbance to emphasize the importance of feedback control. In this case, a $20 \%$ increase in the tube-side inlet mass flow rate is introduced into the simulation settings to simulate the feed disturbance. In the presence of a tube-side feed disturbance, PI control and open-loop control yield significantly different temperature trajectories of the outer reforming tube wall (Fig. 11 and Fig. (16). Unlike feedback control schemes, open-loop control does not possess a self-correcting mechanism, and therefore, open-loop control fails to recognize the presence of the induced tube-side feed disturbance. As a result, open-loop control fails to drive $\bar{x}_{H_{2}}^{\text {outlet }}$ to the desired $\bar{x}_{H_{2}}^{\text {set }}$ (Fig. 17) since it blindly applies the pre-determined outer reforming tube wall temperature trajectory, which is independent from $\bar{x}_{H_{2}}^{\text {outlet }}$. On the contrary, under PI control, $\bar{x}_{H_{2}}^{\text {outlet }}$ is driven to the desired $\bar{x}_{H_{2}}^{\text {set }}$ in the presence of a tube-side feed disturbance within $\sim 308$ seconds. This result demonstrates that the performance of PI control is superior to that of open-loop control (as well as P control) in the presence of a tube-side feed disturbance. Most importantly, the above analysis confirms that it is possible to utilize CFD software to model, design and implement feedback control schemes into a CFD model to form a closed-loop system to study disturbance compensation.

Remark 2. Although the open-loop, P, and PI set-point tracking and disturbance rejection properties are widely known, the purpose of this work is to evaluate the closed-loop properties of the SMR system and, more importantly, to demonstrate that real-time classical and advanced feedback control schemes can be incorpo- 
rated into CFD models, and specifically within the model of the industrial-scale SMR system. P/PI controllers are examples of real-time classical feedback control schemes, while dynamic optimization with integral feedback control (to be presented in detail in the next section) is an example of a real-time advanced feedback control scheme. The simulation results demonstrate the expected dynamic responses, which in turn provides necessary evidence to support the accuracy and effectiveness of the proposed methodology for real-time closed-loop feedback control design based on CFD modeling technology.

\subsection{Integrating dynamic optimization and feedback}

In previous sections, we demonstrate that we can design closed-loop CFD models under feedback control schemes in Ansys Fluent CFD software, and analyze and evaluate the performance of P control and PI control in the presence and absence of a tube-side feed disturbance. In both scenarios, PI control possesses the most enhanced process dynamics and zero-offset in the final $\bar{x}_{\mathrm{H}_{2}}^{\text {outlet }}$, and therefore, it is ranked to have the best performance. Nevertheless, we strive to improve the process dynamics further, and in this regard, we would like to formulate more advanced control schemes, i.e., optimization-based control schemes, to design a closed-loop system in CFD software that drives $\bar{x}_{H_{2}}^{\text {outlet }}$ to the desired $\bar{x}_{H_{2}}^{\text {set }}$. Optimization-based control schemes (e.g., model predictive control - MPC) have been widely used in the HyCO/SMR plants of the gas industry. Successful implementation of optimization-based control of SMR plants can allow more effective set-point tracking and disturbance rejection properties. Therefore, motivated by industrial practical considerations, we devote the remaining section to 
the development of a dynamic optimization and integral feedback control scheme (Fig. 18) which generates the outer reforming tube wall temperature profile.

The majority of approximate models formulated to capture the dominant dynamics of the reforming tube for the purpose of designing optimization-based control schemes such as MPC in industry are data-based models. Hence, we first derive a mathematical model capturing the relationship between $\bar{x}_{H_{2}}^{\text {outlet }}$ and the outer reforming tube wall temperature by utilizing a data-driven modeling technique (previously mentioned in Section 3.3) and CFD simulation results. It is worth emphasizing that all CFD simulation settings from the previous sections are again used in this section. From the CFD simulation result of the step-change of the maximum outer reforming tube wall temperature from $T_{\text {Wall }}^{\max }(0)=1100 \mathrm{~K}$ to $T_{W a l l}^{\max }(t)=1110 \mathrm{~K}$, the dynamic response of $\bar{x}_{H_{2}}^{\text {outlet }}$ exhibits a behavior that can be described by a first-order transfer function indicated by the initial non-zero slope. Hence, the discrete first-order single-input-single-output (SISO) model presented in Eq. 9a below is formulated to represent the transfer function of the process. Then, based on this first-order SISO model, the maximum likelihood estimation (MLE) method Rogers and Steiglitz (1967); Kumar and Varaiya (1986) presented in Eq. $9 b$ is used to identify the appropriate model parameters with a set of outer reforming tube wall temperature and $\bar{x}_{H_{2}}^{\text {outlet }}$ data from the above CFD simulation. The discrete first order SISO model and MLE formulation are presented as follows: 


$$
\begin{aligned}
& y(k)=\frac{B(s)}{A(s)} u(k)+\frac{1}{A(s)} e(k) \\
& \left\{\begin{array}{l}
A(s)=a_{0}+a_{1} s \\
B(s)=b_{0}
\end{array}\right. \\
& \tau(y(k), u(k))=\underset{\theta \in \Theta}{\arg \max }\{y(k) \mid u(k), \theta\}
\end{aligned}
$$

where $y(k)$ and $u(k)$ are the process output and process input respectively, $s$ is an independent variable on the Laplace domain of the transfer function, $k$ is a discrete time variable, $\tau(y(k), u(k))$ is the maximum likelihood estimator, $\theta=\left[\begin{array}{lll}a_{0} & a_{1} & b_{0}\end{array}\right]$ is the parameter vector of the estimated model, and $e(k)$ is assumed to be a value of a white noise function with zero mean and a standard deviation of 1 . Utilizing the aforementioned strategy, $a_{0}, a_{1}$ and $b_{0}$ are estimated to be $2.188 \times 10^{-3}, 1.000$ and $1.764 \times 10^{-5}$ respectively, and therefore, the approximate model that describes the dominant dynamics of the reforming tube is created.

Subsequently, this dynamic model is applied in the Matlab MPC Design Toolbox (see Table 6 for the parameters used) for the calculation of an optimal maximum outer reforming tube wall temperature trajectory, $T_{\text {Wall }}^{\max , p}\left(t_{k}+\Delta t\right)$, based on the desired $\bar{x}_{\mathrm{H}_{2}}^{\text {set }}$ and aforementioned constraints on the outer reforming tube wall temperature (Eqs. $8 \mathrm{c} 8 \mathrm{~d}$ ). Then, based on this reference manipulated input trajectory, the proposed dynamic optimization and integral feedback control scheme is formulated as follows: 


$$
\begin{aligned}
& \left\{\begin{array}{l}
e\left(t_{k}\right)=\bar{x}_{H_{2}}^{\text {set }}-\bar{x}_{H_{2}}^{\text {outlet }}\left(t_{k}\right) \\
u_{I}\left(t_{k}\right)=\frac{1}{\tau_{I}} \int_{t_{0}}^{t_{k}} e(\tau) d \tau \\
T_{W \text { mall }}^{\text {max }}\left(t_{k}+\Delta t\right)= \\
\quad T_{\text {Wall }}^{\text {max }, p}\left(t_{k}+\Delta t\right)+u_{I}\left(t_{k}\right)
\end{array}\right. \\
& T_{\min } \leq T_{\text {Wall }}\left(x, t_{k}+\Delta t\right) \leq T_{\max } \\
& T_{W a l l}\left(x, t_{k}+\Delta t\right)= \\
& {\left[\begin{array}{lll}
x^{4} x^{3} x^{2} x^{1} x^{0}
\end{array}\right]\left[\begin{array}{c}
-0.0221 \\
0.8003 \\
-10.734 \\
64.416 \\
T_{\text {Wall }}^{\max }\left(t_{k}+\Delta t\right)-151.83
\end{array}\right]}
\end{aligned}
$$

where $T_{W a l l}^{\max , p}\left(t_{k}+\Delta t\right)$ is the reference manipulated input trajectory determined at $t_{k}+\Delta t$ from the dynamic optimization using the MPC algorithm in Matlab. At the end of each sampling time interval, a measurement of $\bar{x}_{H_{2}}^{\text {outlet }}$ acquired from

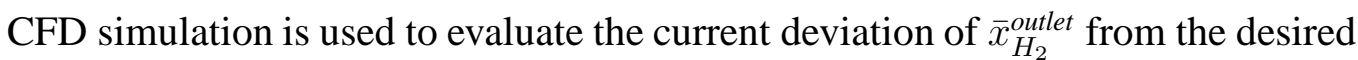
$\bar{x}_{H_{2}}^{\text {set }}$, and the corresponding integral control action, $u_{I}\left(t_{k}\right)$, is determined. Then, $T_{\text {Wall }}^{\text {max }}\left(t_{k}+\Delta t\right)$, obtained from the strategy described in the preceding paragraph, is adjusted by $u_{I}\left(t_{k}\right)$ to yield the predicted $T_{W a l l}^{\max }\left(t_{k}+\Delta t\right)$ as the manipulated input to the closed-loop CFD model.

We compare the performance of the dynamic optimization and integral feedback control scheme with those of PI feedback control and open-loop control based on the two aforementioned criteria for control performance in Sec. 3.2, It is worth mentioning that the primary role of integral control in the dynamic optimization and integral feedback control scheme is to guarantee that $\bar{x}_{H_{2}}^{\text {outlet }}$ can 
Table 6: Matlab MPC Design Toolbox parameters.

\begin{tabular}{ll}
\hline Parameter & Value \\
\hline Input weight & 0.00 \\
Output weight & 1.00 \\
Overall estimator gain & 0.5 \\
Control step & 0.1 seconds \\
Prediction horizon & 2000 steps \\
Control horizon & 20 steps \\
\hline
\end{tabular}

always attain the desired $\bar{x}_{H_{2}}^{\text {set }}$ even in the presence of a tube-side feed disturbance. In the case of set-point tracking control under a tube-side feed disturbance, both the dynamic optimization and integral feedback control scheme and the PI control scheme can drive the system to the desired set-point; however, the dynamic optimization and integral feedback control scheme predicts a slightly different outer reforming tube temperature trajectory than that predicted by PI control as shown in Fig. 20. Specifically, the maximum outer reforming tube wall temperature of the closed-loop system under the dynamic optimization with integral feedback control scheme is initially maintained at $1200 \mathrm{~K}$ for nearly 40 seconds as shown in Fig. 19, which speeds up the process dynamic response. Based on the metric that we previously defined in this study, it only takes $\sim 33$ seconds for $\bar{x}_{H_{2}}^{\text {outlet }}$ to first achieve the desired $\bar{x}_{H_{2}}^{\text {set }}$ under the dynamic optimization with integral feedback control scheme as shown in Fig. 20, corresponding to an improvement of $89.3 \%$ when compared with the system under PI control for which it takes $\sim 308$ seconds. Nevertheless, the aggressive manipulated input generated by the dynamic optimization with integral feedback control scheme also causes the oscillation of 
$\bar{x}_{H_{2}}^{\text {outlet }}$ around the desired $\bar{x}_{H_{2}}^{\text {set }}$. Therefore, it is important to notice that although the dynamic optimization with integral feedback control scheme allows $\bar{x}_{H_{2}}^{\text {outlet }}$ to advance to the desired $\bar{x}_{\mathrm{H}_{2}}^{\text {set }}$ much faster than PI control, it ultimately requires a longer duration to reach the optimized steady-state conditions and results in oscillation; therefore, its performance is comparable with that of PI feedback control.

Remark 3. As discussed above, the PI controller is easier to implement than the dynamic optimization with integral feedback control scheme but it does not use an explicit plant model, and as a result, the closed-loop response is slower because the tuning was chosen to avoid high sensitivity to disturbances. Further, the overall PI closed-loop response was not improved for other tunings investigated that reasonably avoided this sensitivity (another result is discussed below that confirms the sufficiency of the PI controller with the chosen tuning by demonstrating its robustness to various disturbances). However, the dynamic optimization with integral feedback control scheme achieves a faster closed-loop response than the PI controller with the knowledge of the process dynamics; plant-model mismatch, integral action, and the effect of the disturbance may contribute to the oscillations in the output trajectory. The comparison results demonstrated that both control schemes force the output to track the set-point and deal well with disturbances. As noted, the sufficiency of the chosen PI controller tuning is further validated by testing the closed-loop response of $\bar{x}_{\mathrm{H}_{2}}^{\text {outtet }}$ to a different disturbance than that utilized in the results presented above. An additional disturbance in the tubeside feed, i.e., the temperature of the tube-side feed is increased by $10 \%$ from its nominal value, is induced to validate the robustness of the PI controller against 
different potential disturbances. The simulation results including the control action (Fig. 21) and corresponding process response (Fig. 22) generated by the PI controller to counteract the tube-side feed temperature disturbance are similar to those of the previous case in Sec. 3.3 when the induced disturbance is the tubeside feed mass flow rate. Specifically, $\bar{x}_{\mathrm{H}_{2}}^{\text {outlet }}$ is successfully driven to the desired $\bar{x}_{H_{2}}^{\text {set }}$ in the presence of a tube-side feed temperature disturbance within $\sim 200$ seconds.

\section{Conclusions}

This work initially focused on demonstrating that CFD software can be employed to create a detailed CFD model of an industrial-scale steam methane reforming tube, and subsequently focused on the design and implementation of feedback control schemes into the CFD model. The simulation results of the reforming tube CFD model simulating the transport and chemical reaction phenomena with an approximate representation of the catalyst packing matched well with the available industrial plant data. The closed-loop CFD simulation results demonstrated that the proposed PI control scheme and a control scheme combining dynamic optimization and integral feedback control could drive the value of $\bar{x}_{H_{2}}^{\text {outlet }}$ to a desired $\bar{x}_{H_{2}}^{\text {set }}$, and significantly improve the process dynamics compared to that under open-loop control. In the case of set-point tracking control under a tube-side feed disturbance, the dynamic optimization with integral feedback control scheme calculates a more aggressive outer reforming tube wall temperature trajectory compared to that calculated by PI control but both control systems drive 
809 the average outlet hydrogen mole fraction to the set-point.

\section{5. Acknowledgements}

811 Financial support from the Department of Energy is gratefully acknowledged. 


\section{References}

Adams, T.A., Barton, P.I., 2009. A dynamic two-dimensional heterogeneous model for water gas shift reactors. International Journal of Hydrogen Energy 34, 8877-8891.

Aeronautics, N., Administration, S., . NASA's viscous grid spacing calculator. http://geolab.larc.nasa.gov/APPS/YPlus/ .

Baburić, M., Duić, N., Raulot, A., Coelho, P.J., 2005. Application of the conservative discrete transfer radiation method to a furnace with complex geometry. Numerical Heat Transfer, Part A: Applications 48, 297-313.

Behnam, N., Dixon, A.G., Wright, P.M., Nijemeisland, M., Stitt, E.H., 2012. Comparison of CFD simulations to experiment under methane steam reforming reacting conditions. Chemical Engineering Journal 207-208, 690-700.

Beyer, F., Brightling, J., Farnell, P., Foster, C., 2005. Steam reforming - 50 years of development and the challenges for the next 50 years, in: AIChE 50th Annual Safety in Ammonia Plants and Related Facilities Symposium, Toronto, Canada.

Calis, H.P.A., Nijenhuis, J., Paikert, B.C., Dautzenberg, F.M., van den Bleek, C.M., 2001. CFD modelling and experimental validation of pressure drop and flow profile in a novel structured catalytic reactor packing. Chemical Engineering Science 56, 1713-1720.

Davis, J.R., 2000. Alloy Digest Sourcebook: Stainless Steels (Alloy Digest). ASM International, Materials Park, $\mathrm{OH}$. 
Dixon, A.G., 2014. CFD study of effect of inclination angle on transport and reaction in hollow cylinder catalysts. Chemical Engineering Research and Design 92, 1279-1295.

Dybkjaer, I., 1995. Tubular reforming and autothermal reforming of natural gas an overview of available processes. Fuel Processing Technology 42, 85-107.

Ergun, S., Orning, A.A., 1949. Fluid flow through randomly packed columns and fluidized beds. Industrial \& Engineering Chemistry 41, 1179-1184.

Froment, G.F., Bischoff, K.B., 1990. Chemical reactor analysis and design. Wiley, New York.

Guardo, A., Coussirat, M., Larrayoz, M.A., Recasens, F., Egusquiza, E., 2004. CFD flow and heat transfer in nonregular packings for fixed bed equipment design. Industrial \& Engineering Chemistry Research 43, 7049-7056.

Han, Y., Xiao, R., Zhang, M., 2006. Combustion and pyrolysis reactions in a naphtha cracking furnace. Chemical Engineering \& Technology 30, 112-120.

Inc., A., Nov. 2013a. ANSYS Fluent Theory Guide 15.0 .

Inc., A., Nov. 2013b. ANSYS Fluent User's Guide 15.0 .

Jeong, J.H., Lee, J.W., Seo, D.J., Seo, Y., Yoon, W.L., Lee, D.K., Kim, D.H., 2006. $\mathrm{Ru}$-doped Ni catalysts effective for the steam reforming of methane without the pre-reduction treatment with $\mathrm{H}_{2}$. Applied Catalysis A: General 302, 151-156. 
Jones, W.P., Launder, B.E., 1972. The prediction of laminarization with a twoequation model of turbulence. International Journal of Heat and Mass Transfer $15,301-314$.

Kumar, P.R., Varaiya, P., 1986. Stochastic systems: estimation, identification and adaptive control. Prentice-Hall, Inc., Upper Saddle River, NJ.

Latham, D., 2008. Masters Thesis: Mathematical Modeling of An Industrial Steam Methane Reformer. Queen's University.

Launder, B.E., Sharma, B.I., 1974. Application of the energy dissipation model of turbulence to the calculation of flow near a spinning disc. Letters in Heat and Mass Transfer 1, 131-137.

$\begin{array}{cccccc}\text { Matthey, } & \text { J., } & \text { Johnson } & \text { Matthey } & \text { Catalysts } & \text { De- } \\ \text { livering } & & \text { world-class } & \text { hydrogen } & \text { plant } & \text { performance. }\end{array}$ http://www.jmcatalysts.cn/en/pdf/HydrogenTechBrochFeb2007.pdf .

McGreavy, C., Newmann, M.W., 1969. Development of a mathematical model of a steam methane reformer, in: Institution of Electrical Engineering, Conference on the Industrial Applications of Dynamic Modelling, Durham, NC.

Noor, M.M., Wandel, A.P., Yusaf, T., 2013. Detail guide for CFD on the simulation of biogas combustion in bluff-body mild burner, in: International Conference on Mechanical Engineering Research, Kuantan, Malaysia.

Othmer, K., . Kirk-Othmer Encyclopedia of Chemical Technology. John Wiley and Sons Inc. 
Pantoleontos, G., Kikkinides, E.S., Georgiadis, M.C., 2012. A heterogeneous dynamic model for the simulation and optimisation of the steam methane reforming reactor. International Journal of Hydrogen Energy 37, 16346-16358.

Rogers, A.E., Steiglitz, K., 1967. Maximum likelihood estimation of rational transfer function parameters. IEEE Transactions on Automatic Control 12, 594-597.

Rostrup-Nielsen, J.R., 1984. Catalysis: Science and Technology. Springer-Verlag, Berlin, Germany. chapter Catalytic Steam Reforming. pp. 1-117.

Stefanidis, G.D., Merci, B., Heynderickx, G.J., Marin, G.B., 2006. CFD simulations of steam cracking furnaces using detailed combustion mechanisms. Computers \& Chemical Engineering 30, 635-649.

Uriz, I., Arzamendi, G., Diéguez, P.M., Gandía, L.M., 2013. Computational fluid dynamics as a tool for designing hydrogen energy technologies, in: Renewable Hydrogen Technologies: Production, Purification, Storage, Applications and Safety. Elsevier, Oxford, UK, pp. 401-435.

Wesenberg, M.H., Svendsen, H.F., 2007. Mass and heat transfer limitations in a heterogeneous model of a gas-heated steam reformer. Industrial \& Engineering Chemistry Research 46, 667-676.

Xu, J., Froment, G.F., 1989. Methane steam reforming, methanation and watergas shift: I. intrinsic kinetics. AIChE Journal 35, 88-96. 


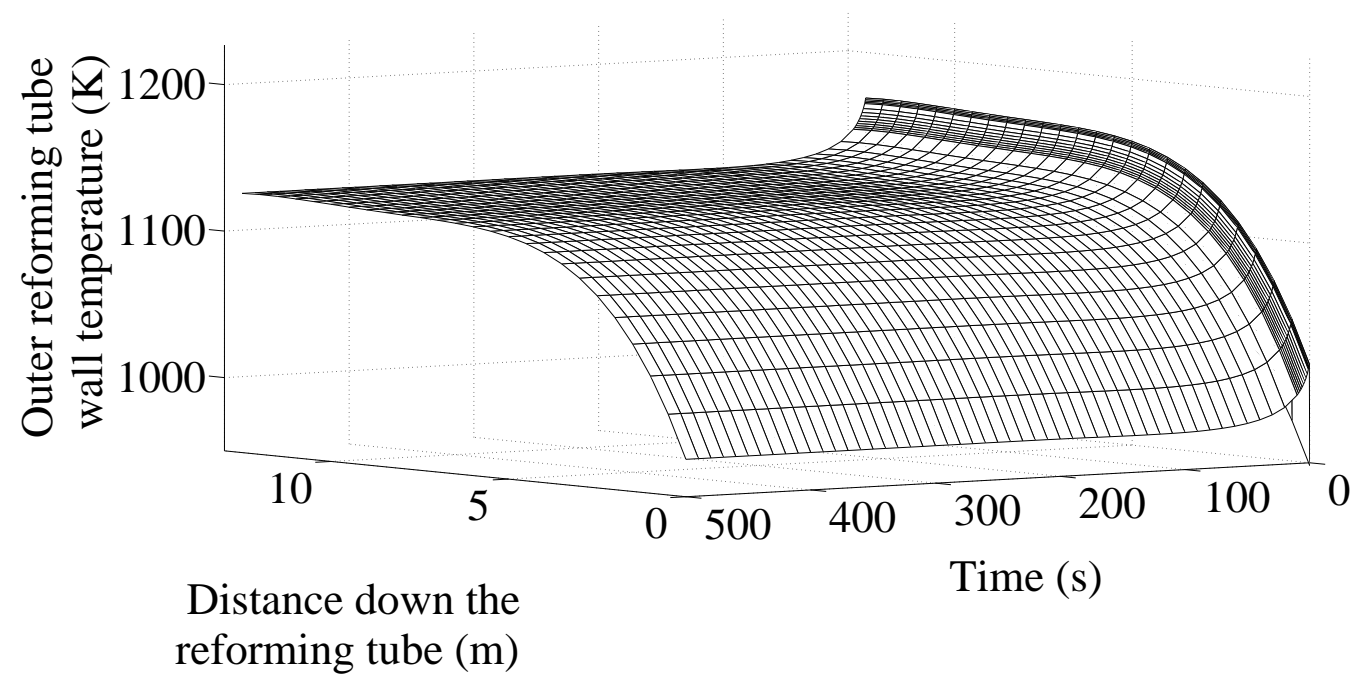

(a)

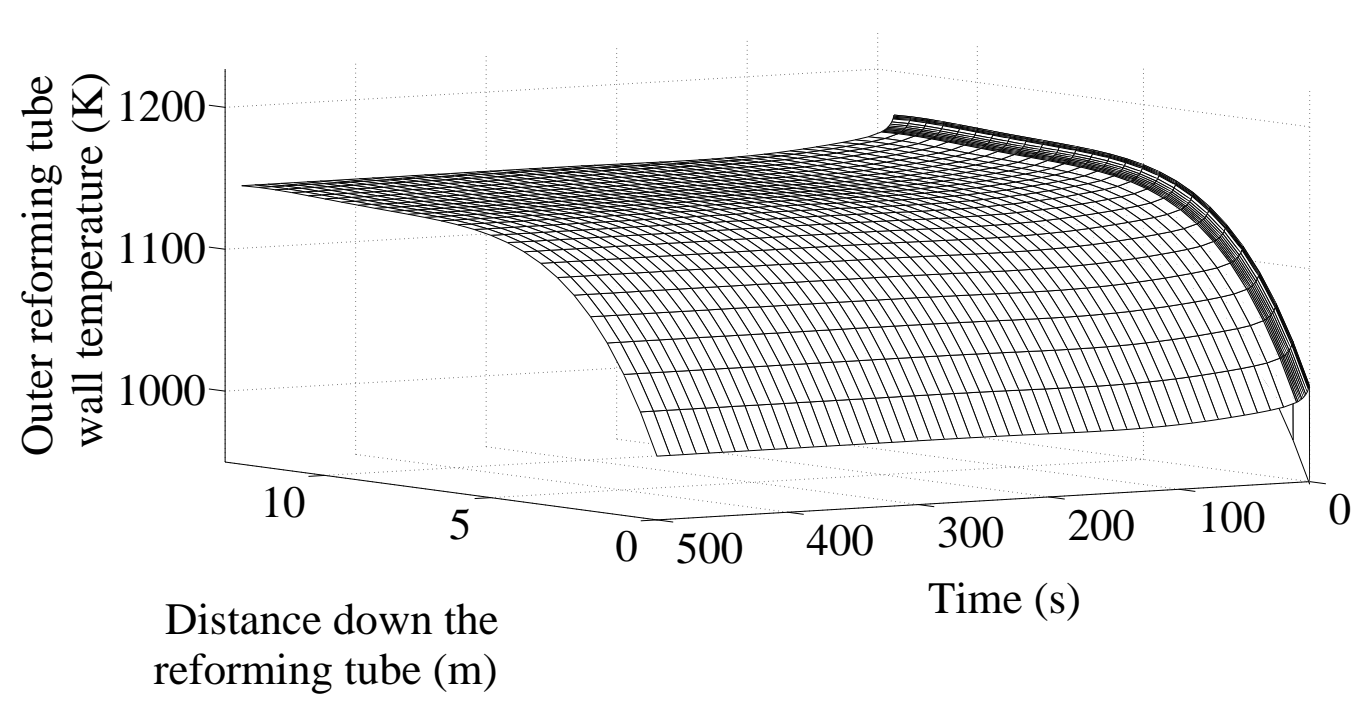

(b)

Figure 14: The outer reforming tube wall temperature profile trajectory in the absence of a tubeside feed disturbance under P control shown in Fig.14(a) and under PI control shown in Fig.14(b) with $\bar{x}_{H_{2}}^{s e t}=0.465$. 


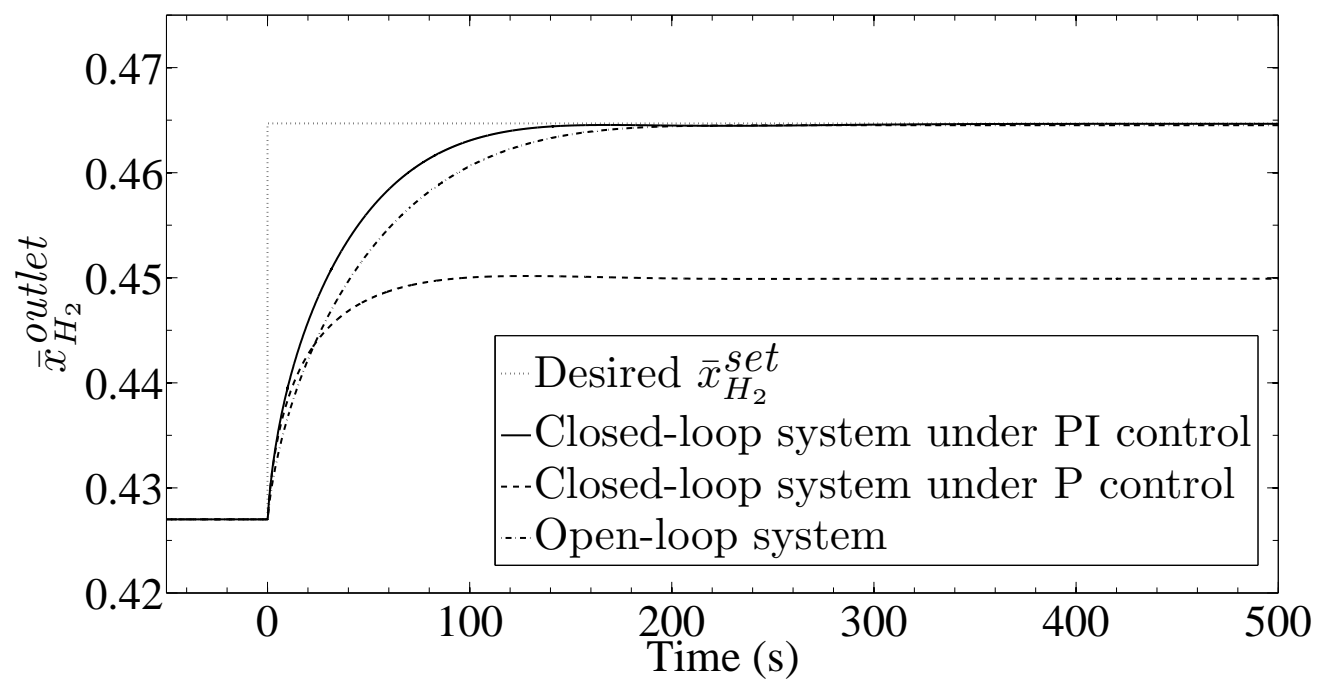

Figure 15: The propagation of $\bar{x}_{H_{2}}^{\text {outlet }}$ with time in the absence of a tube-side feed disturbance under P control (solid line) and under PI control (dashed line). The open-loop system response (dashed-dotted line) is also included for a wall temperature profile for which $\bar{x}_{H_{2}}^{\text {set }}=0.465$.

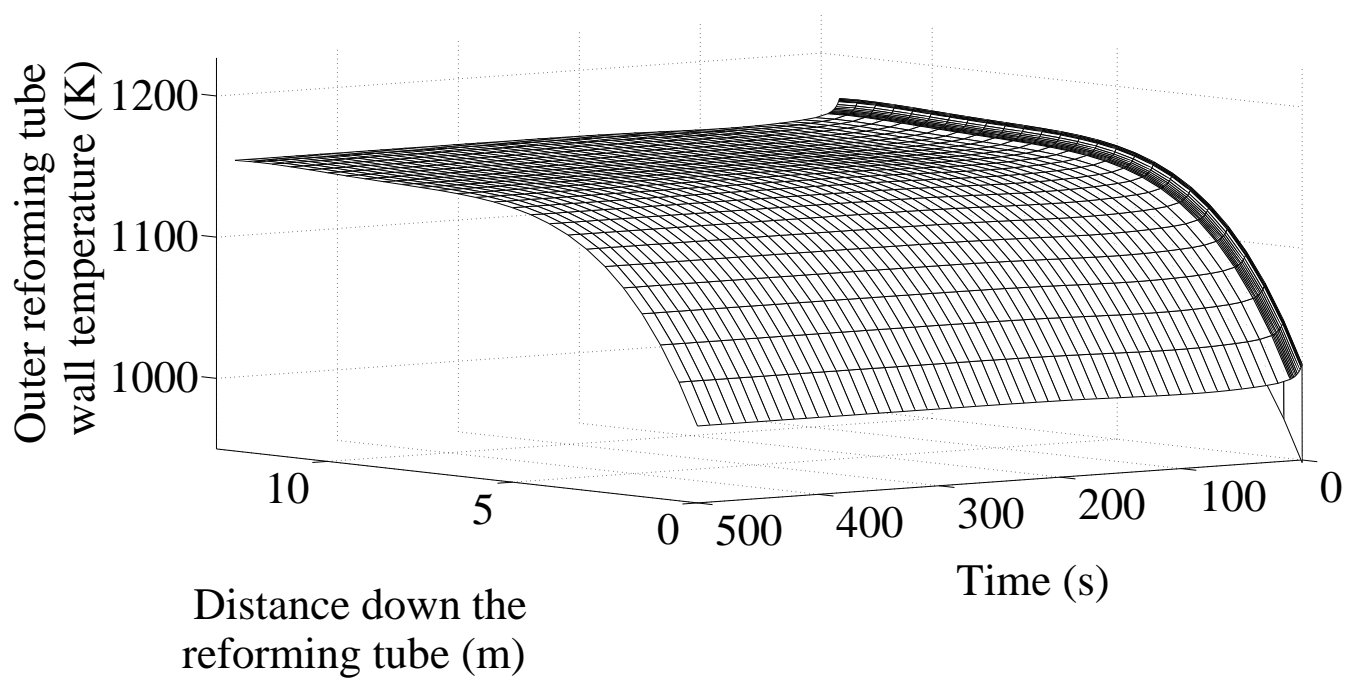

Figure 16: The outer reforming tube wall temperature profile trajectory in the presence of a disturbance in the tube-side feed ( $20 \%$ increase in tube-side mass flow rate) under PI control with $\bar{x}_{H_{2}}^{\text {set }}=0.465$. 


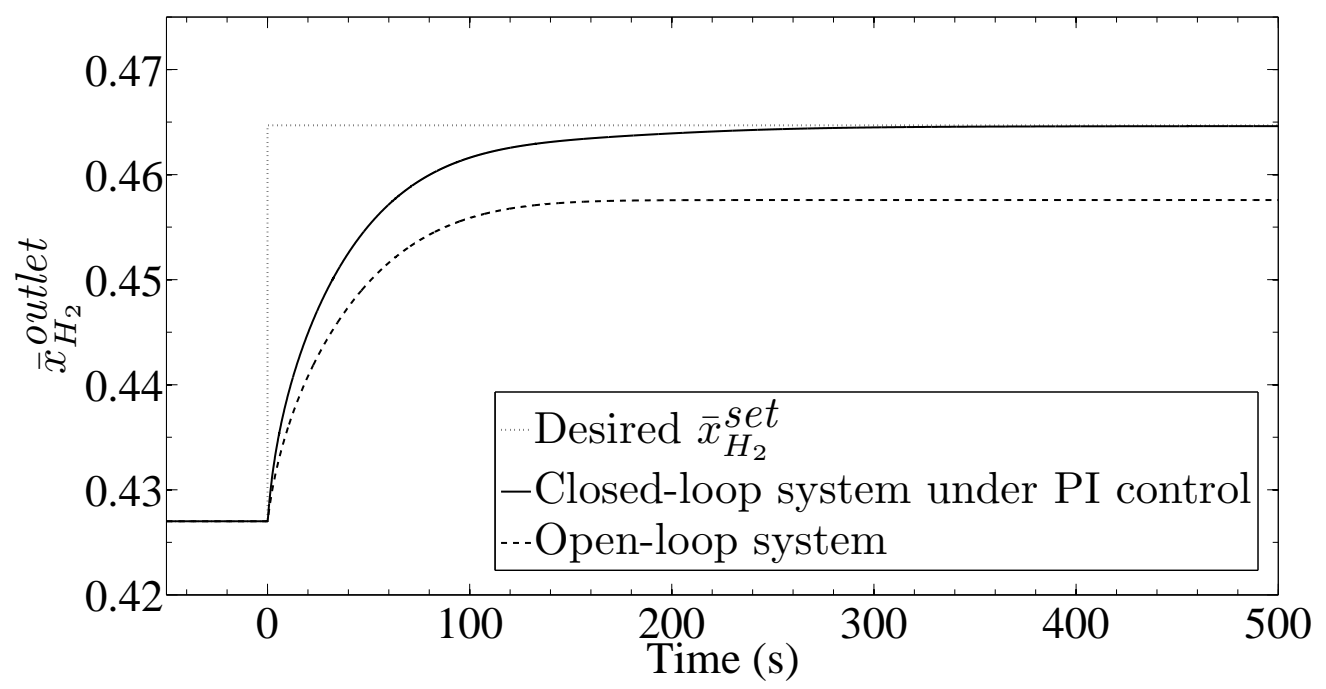

Figure 17: The propagation of $\bar{x}_{H_{2}}^{\text {outlet }}$ with time in the presence of a disturbance in the tube-side feed ( $20 \%$ increase in tube-side mass flow rate) under PI control (solid line) and under open-loop control (dashed line) for a wall temperature profile for which $\bar{x}_{H_{2}}^{\text {set }}=0.465$. 


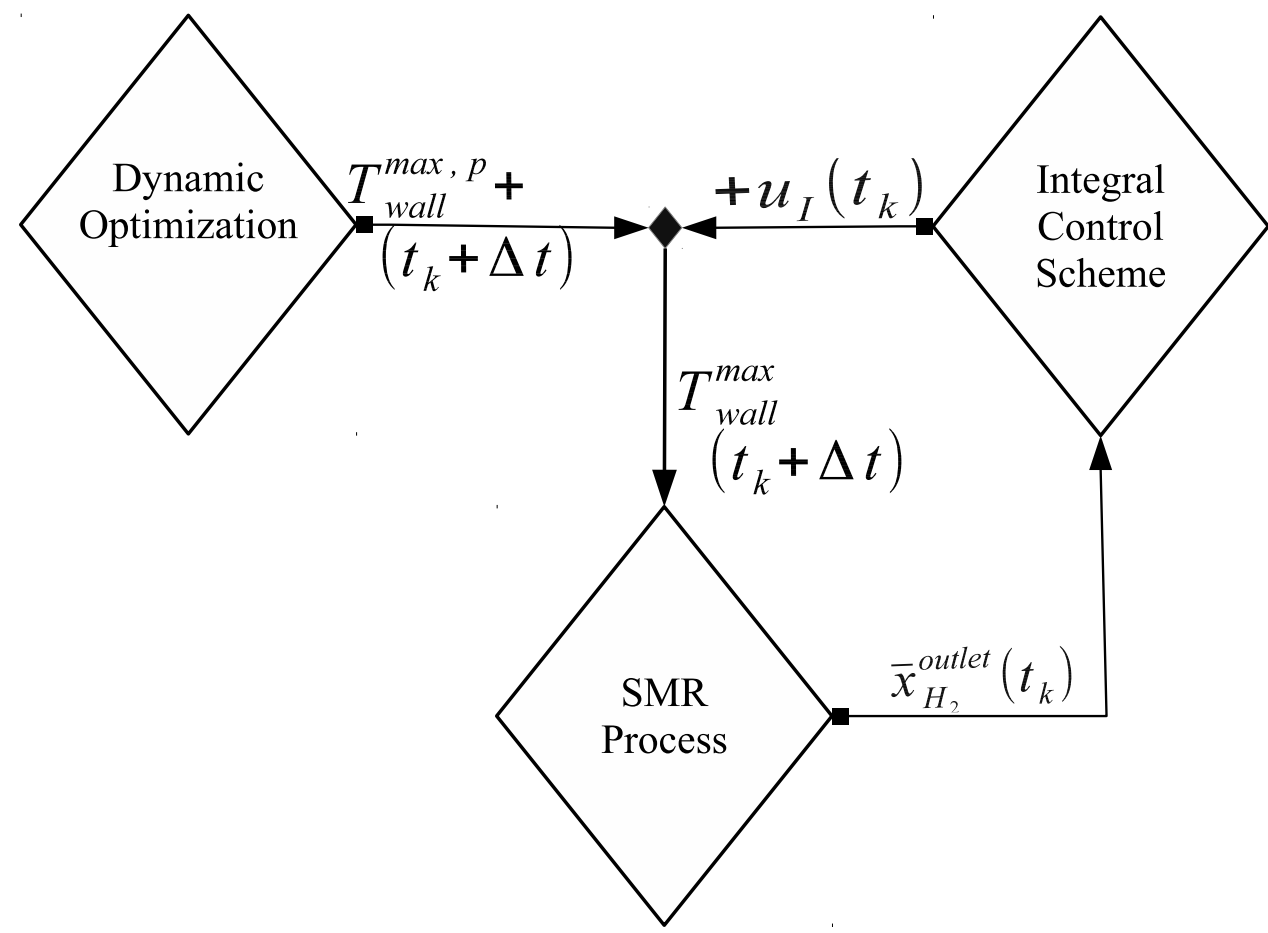

Figure 18: Dynamic optimization and integral feedback control scheme, where the manipulated input at $t_{k}+\Delta t$ is computed based on the reference manipulated input profile at $t_{k}+\Delta t$ and the integral control action at $t_{k}$, which is described in Eq.10. 


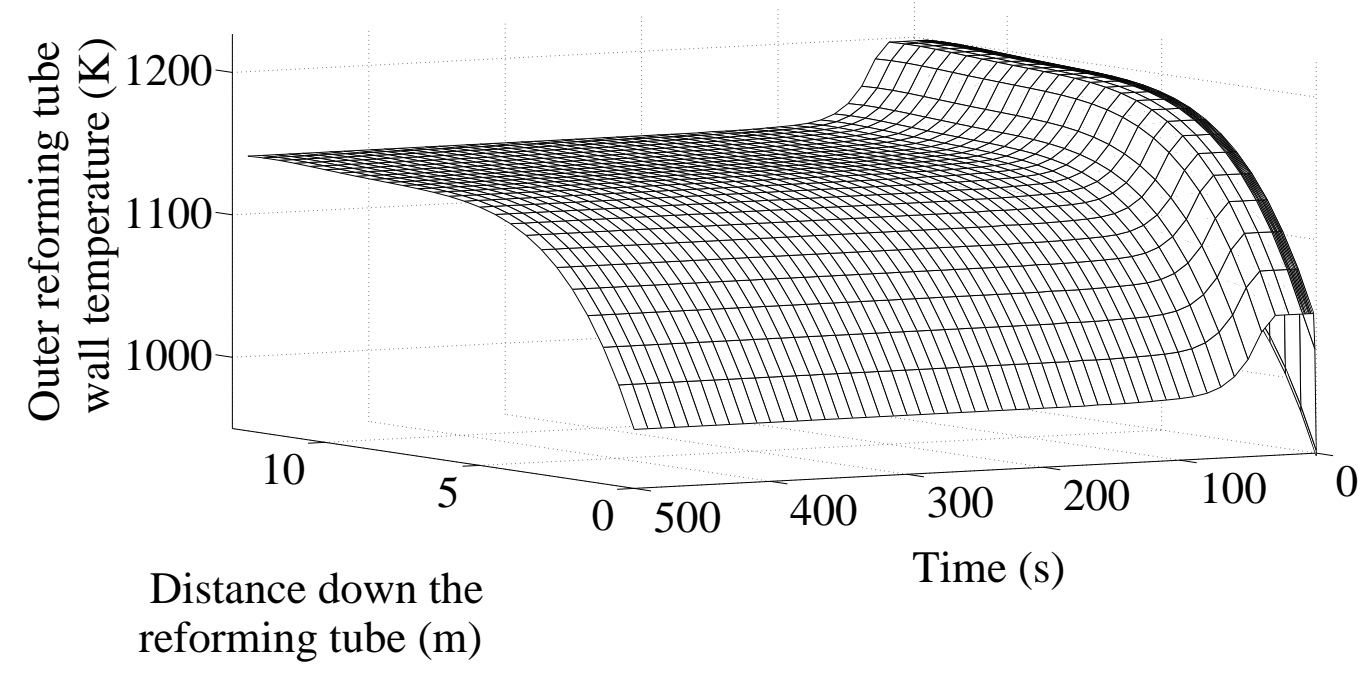

Figure 19: The outer reforming tube wall temperature profile trajectory in the presence of a tubeside feed disturbance ( $20 \%$ increase in tube-side mass flow rate) under the dynamic optimization with integral feedback control scheme with $\bar{x}_{H_{2}}^{s e t}=0.465$.

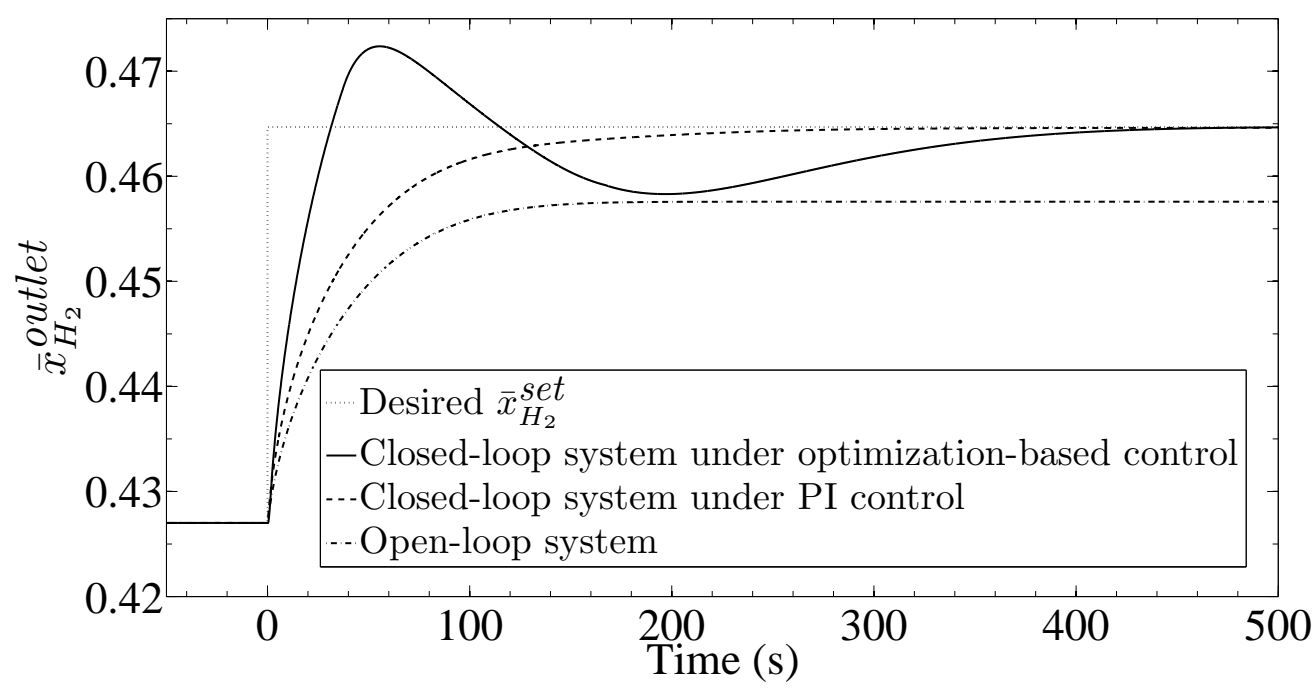

Figure 20: The propagation of $\bar{x}_{H_{2}}^{\text {outlet }}$ with time in the presence of a tube-side feed disturbance (20\% increase in tube-side mass flow rate) under the dynamic optimization with integral feedback control scheme (solid line) and under PI control (dashed line). The open-loop system response (dashed-dotted line) is also included for a wall temperature profile for which $\bar{x}_{H_{2}}^{\text {set }}=0.465$. 


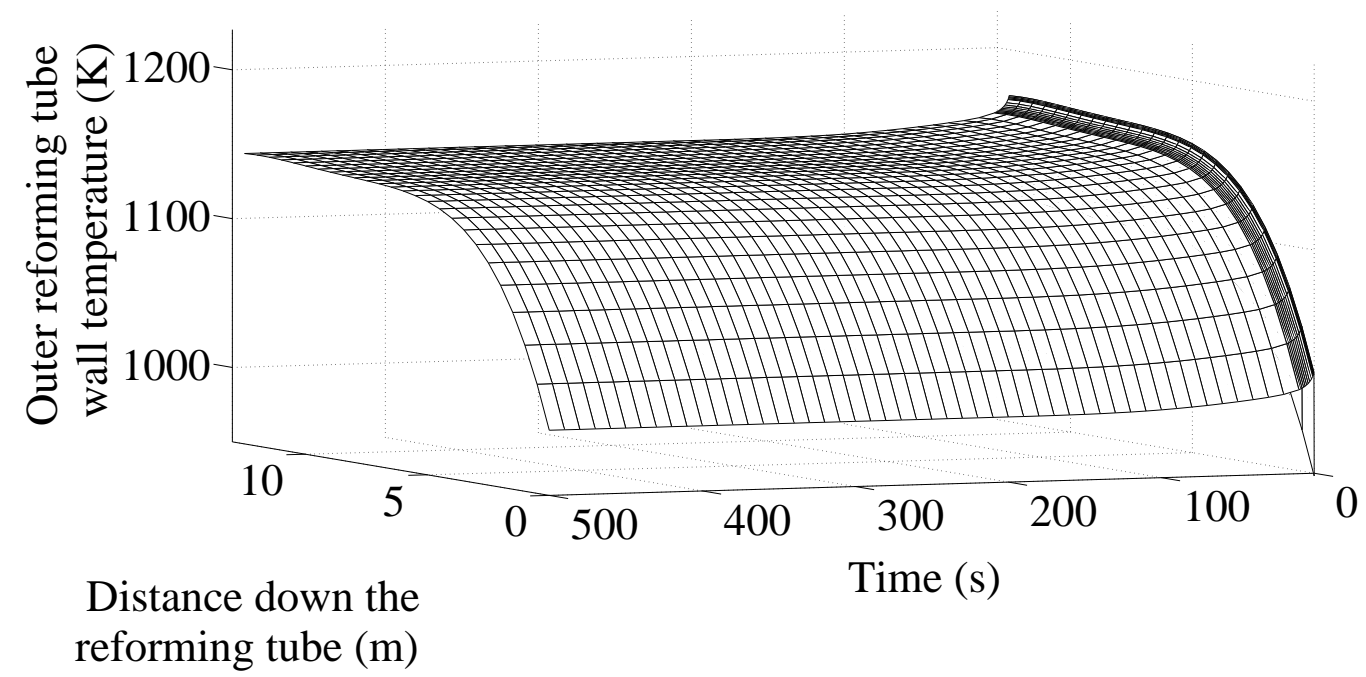

Figure 21: The outer reforming tube wall temperature profile trajectory in the presence of a disturbance in the tube-side feed temperature under PI control with $\bar{x}_{H_{2}}^{\text {set }}=0.465$.

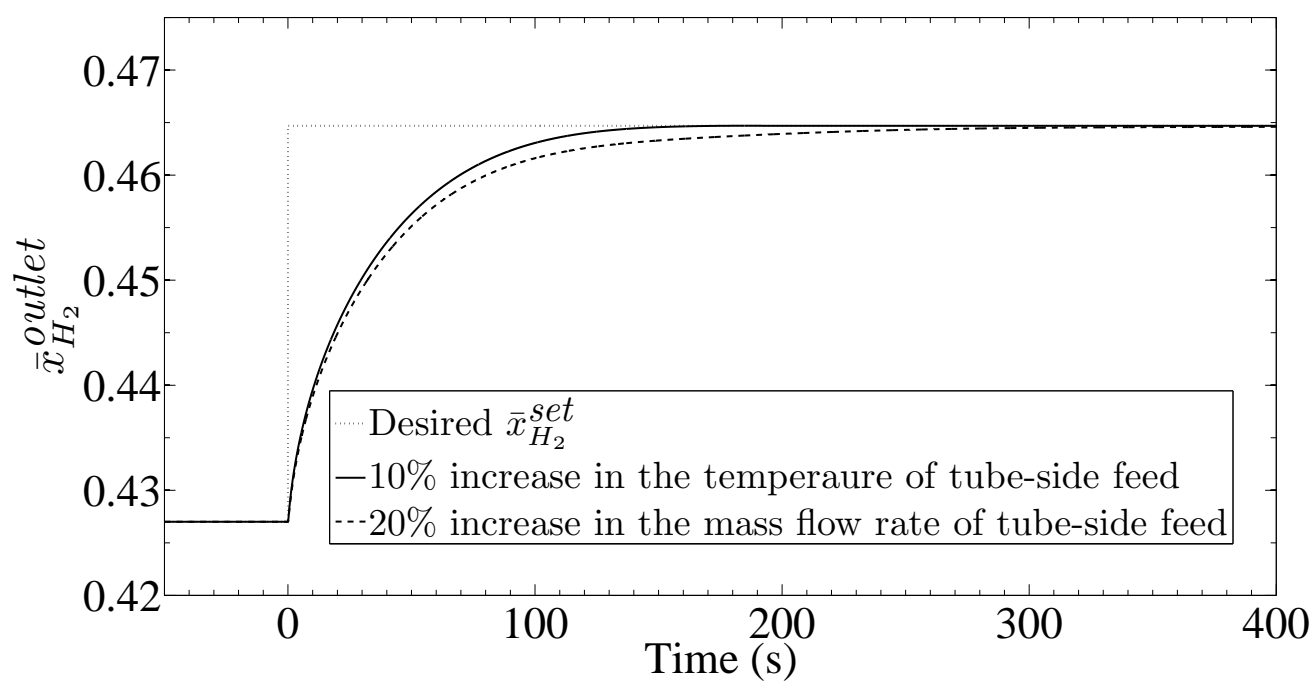

Figure 22: The propagation of $\bar{x}_{H_{2}}^{\text {outlet }}$ with time in the presence of a disturbance in the tube-side feed temperature (solid line) and in the presence of a disturbance in the tube-side feed mass flow rate (dashed line) under PI control for a wall temperature profile for which $\bar{x}_{H_{2}}^{\text {set }}=0.465$. 